\title{
Du discours dynastique au corps social. Retour sur la terminologie des groupes aristocratiques incas de Cuzco
}

Dynastic discourse and social groups. A reexamination of the terminology of inca aristocratic groups in Cuzco

Discurso dinástico y cuerpo social. Revisando la terminología de los grupos

aristocráticos incas del Cuzco

\section{Laurent Segalini}

\section{OpenEdition \\ Journals}

Édition électronique

URL : https://journals.openedition.org/jsa/12603

DOI : 10.4000/jsa. 12603

ISSN : 1957-7842

\section{Éditeur}

Société des américanistes

Édition imprimée

Date de publication : 15 septembre 2013

Pagination : 45-75

ISSN : 0037-9174

Référence électronique

Laurent Segalini, «Du discours dynastique au corps social. Retour sur la terminologie des groupes aristocratiques incas de Cuzco », Journal de la Société des américanistes [En ligne], 99-1 | 2013, mis en ligne le 01 mars 2016, consulté le 04 septembre 2022. URL : http://journals.openedition.org/jsa/ 12603 ; DOI : https://doi.org/10.4000/jsa.12603 


\title{
DU DISCOURS DYNASTIQUE AU CORPS SOCIAL. RETOUR SUR LA TERMINOLOGIE DES GROUPES ARISTOCRATIQUES INCAS DE CUZCO
}

\author{
Laurent SEGALINI *
}

L'existence à Cuzco de groupes sociaux censés rassembler les descendants des souverains incas successifs constitue une donnée essentielle des différents modèles d'interprétation de l'histoire dynastique élaborés depuis plusieurs décennies, opposant les tenants d'une interprétation historique à ceux d'une conception mythique des récits et de leurs protagonistes royaux. À partir d'un réexamen du corpus des sources classiques et d'une documentation inédite, le présent article propose de reconsidérer certains aspects de la structure sociale du Cuzco préhispanique et, notamment, l'homogénéité supposée des groupes aristocratiques. S'offre ainsi l'opportunité d'une redéfinition partielle du cadre du débat sur la nature du récit dynastique inca. [Mots-clés : Incas, Cuzco, structure sociale, panaca, ayllu.]

Dynastic discourse and social groups. A reexamination of the terminology of inca aristocratic groups in Cuzco. The existence in Cuzco of social groups supposedly uniting the descendants of successive Inca sovereigns constitutes an essential datum for the various interpretative models of Inca dynastic history elaborated in the last decades, opposing those who defend an historical interpretation to those with a mythical conception of the narratives and their protagonists. Based on a renewed scrutiny of the classical sources as well as on original documentation, this article aims to reconsider some aspects of the social structure of prehispanic Cuzco, especially the supposed homogeneity of the aristocratic groups. At the same time, it provides an opportunity to redefine partially the framework of the discussion about the nature of Inca dynastic narratives. [Key words : Incas, Cuzco, social structure, panaca, ayllu.]

Discurso dinástico y cuerpo social. Revisando la terminología de los grupos aristocráticos incas del Cuzco. La existencia en el Cuzco de grupos sociales que supuestamente juntaban los descendientes de los soberanos incas sucesivos, constituye un dato fundamental para los modelos interpretativos de la historia dinástica elaborados en las últimas décadas. Ellos oponen los partidarios de una interpretación histórica a los de

* Université Sorbonne Nouvelle Paris 3, Paris [laursega@yahoo.fr].

Journal de la Société des Américanistes, 2013, 99-1, pp. 45-75. (C) Société des Américanistes. 
una concepción mítica de los relatos y de sus protagonistas reales. Partiendo de un nuevo examen del corpus de las fuentes clásicas así como de una documentación inédita, el presente artículo propone reconsiderar ciertos aspectos de la estructura social del Cuzco prehispánico, particularmente la supuesta homogeneidad de los grupos aristocráticos. Así se brinda la oportunidad de precisar el marco del debate sobre la naturaleza del relato dinástico inca. [Palabras claves : incas, Cuzco, estructura social, panacas, ayllus.]

\section{INTRODUCTION}

Le prestige dont jouit l'empire inca (Tawantinsuyu) dans l'imaginaire occidental depuis le $\mathrm{XVI}^{\mathrm{e}}$ siècle contraste avec la carence de connaissances positives sur certains de ses aspects fondamentaux, en premier lieu une caractérisation précise du récit dynastique recueilli par les chroniqueurs espagnols. Coulé dans le moule de l'historiographie médiévale et renaissante, il narre la geste de douze souverains, depuis l'établissement à Cuzco du héros fondateur Manco Capac jusqu'à la guerre de succession opposant Huascar et Atahuallpa, réglée par l'arrivée des Européens. Or, si l'historicité des trois derniers souverains - dont la mémoire indigène conservait le souvenir - ne semble faire aucun doute, les règnes antérieurs restent plongés dans une pénombre où des faits historiquement plausibles se mêlent inextricablement à des données d'un autre ordre. Le contenu problématique de la tradition orale cuzquénienne divise la communauté scientifique : tandis que certains s'accordent à y voir le compte rendu d'une réalité historique peut-être altérée par le mythe ou retouchée par le pouvoir politique (Rostworowski 1988 ; Julien 2000 ; Rowe 2003b), d'autres la considèrent comme l'expression mythique de réalités sociales, politiques, rituelles, ou d'autres aspects du savoir local (astronomique, calendaire, agricole) historicisés par les chroniqueurs espagnols (Urton 1990 ; Zuidema 1995 [1964]; Pease 1995, pp. 71-78). Enfin certains, tout en admettant le caractère mythique des récits, acceptent néanmoins l'historicité de la dynastie royale qui en est l'objet (Duviols 1979a et 1997).

La radicalité des postures théoriques s'explique notamment par une spécificité cuzquénienne qui leur a servi d'argument commun : l'existence de groupes sociaux censés rassembler les descendants des différents souverains. Dès lors, tandis que les uns peuvent arguer de l'historicité des souverains dynastiques par l'existence de leurs descendants, les autres se fondent sur l'étroite imbrication de l'histoire dynastique dans l'organisation sociale pour montrer que la première n'a été que la projection de la seconde. Zuidema considère, pour sa part, que le dualisme de l'organisation cuzquénienne (Hanan Cuzco « Haut Cuzco »/Urin Cuzco « Bas Cuzco ») exclut une constitution progressive sur douze générations et suppose, au contraire, l'existence simultanée de l'ensemble des groupes sociaux 
dont les ancêtres n'ont pu être alors que des représentants symboliques (Zuidema 1986, pp. 51-66, 2010, p. 421). À quoi Julien (2000, pp. 7-9) objecte qu'une telle position revient à nier l'historicité pourtant bien attestée de certains souverains, sans par ailleurs tenir compte de la possibilité de changements structurels consécutifs à l'expansion de l'état inca. Un débat qui illustre bien les difficultés que soulève l'intrication de l'histoire dynastique et de la structure sociale : objet d'interprétations multiples, souvent contradictoires, la réalité cuzquénienne ne semble jamais s'y résoudre totalement. En l'état actuel des connaissances, l'infrastructure sociale constitue donc le seul point d'approche concret, primant sur les données problématiques de l'histoire dynastique. Toutefois, du fait du peu d'éléments explicites fournis par les chroniques, les différentes conceptions de l'organisation sociale s'alimentent nécessairement peu ou prou d'une interprétation de l'histoire dynastique elle-même, au risque d'assurer à terme la primauté des postulats théoriques sur l'analyse des faits bruts.

Entre une conception envisageant la société cuzquénienne comme le produit mécanique d'une histoire invérifiable, en négligeant les indicateurs de stabilité structurelle, et une autre qui voit dans le récit dynastique l'expression de réalités sociales intemporelles, quitte à nier l'historicité des ancêtres de certains groupes sociaux, il subsiste un espace pour d'autres solutions. Il paraît alors souhaitable de réexaminer les fondements d'une alternative qui achoppe visiblement sur une réalité plus complexe, et notamment l'effectivité de l'imbrication entre le récit dynastique et la structure sociale telle qu'elle se trouve représentée dans les sources. Le présent article vise à renouveler le débat en s'attachant à des données jusqu'alors sous-estimées ainsi qu'à une documentation d'archives inédite qui incitent à reconsidérer certains aspects de la structure sociale du Cuzco préhispanique. Il s'agit en particulier de questionner l'homogénéité des groupes aristocratiques censés descendre d'un ancêtre royal, notamment à la lumière de certains détails suggestifs de la terminologie qui leur est associée.

\section{AYLLU ET PANACA (AYLLU)}

\section{L'apport des sources}

Il faut attendre principalement la fin des années 1550 et surtout les années 1560-1570, période de création des premières paroisses puis de l'encadrement de la population cuzquénienne par les « réductions » tolédanes, pour voir mentionner dans les sources la liste complète des dix groupes aristocratiques cuzquéniens. En écartant du corpus les multiples emprunts identifiables ${ }^{1}$, on compte au final cinq sources théoriquement indépendantes : la Apologética historia sumaria de Las Casas (1967 [1562-1564]), probablement fondée sur une information de 
Santo Tomás ${ }^{2}$ ); la Historia índica de Sarmiento de Gamboa, résultant d'une investigation menée à Cuzco en 1572 (2001 [1572]); les Fábulas y ritos de los incas de Molina (1989 [1575]), synthétisant un matériau recueilli auprès de ses paroissiens indigènes ; la declaración de los quipocamayos (Declaración 1920 [1608] [1542]), provenant d'une enquête menée en 1542 par le vice-roi Vaca de Castro, réutilisée plus tard par un certain Fray Antonio (Duviols 1979b); enfin la Relación de las huacas (1559-1561), document anonyme mis à profit par Cobo dans son Historia del Nuevo Mundo (1956 [1653]) et couramment attribué à Polo de Ondegardo (Bauer 2000, p. 22 ; Zuidema 2010, p. 13) (Figure 1).

\begin{tabular}{|c|c|c|c|c|}
\hline Declaración & Relación & Las Casas & $\begin{array}{l}\text { Sarmiento } \\
\text { de Gamboa }\end{array}$ & Molina \\
\hline$(1608$ [1542]) & $(1559-1561)$ & $(1562-1564)$ & (1572) & $(1575)$ \\
\hline Chima panaca & Chimapanaca & Chima panaca & Chima panaca ayllo & Chinapanaca aillu \\
\hline Raorao panaca & Rauraua panaca* & Raurau panaca & Raura panaca & Raurapanaca aillu \\
\hline Ayllo Guguayuin & Haguayni & Haguayni & Avayni panaca ayllo & Yahuaymin ayllu \\
\hline Ayllo Uscamayta & Usca Mayta & Uzcamayta & Usca Mayta panaca ayllo & Uscaymata ayllu \\
\hline Ayllo Apomayta & Apu Mayta & Apomaytha & Apo Mayta panaca ayllo & Apo may ho ayllu \\
\hline Ayllo Vica Quirao & Vicaquirao & Vicaquirau panaca & Vicaquirau panaca ayllo & Vicaquicao \\
\hline Aucayllo panaca & Aucailli panaca & Aucaylli panaca & Aucaylli panaca & Aucaylli ayllo \\
\hline Ayllo Sucsu panaca & Cubçu pañaca ayllu & Cuçco panaca & Coçco panaca ayllo & Cuscupanaca ayllu \\
\hline Ayllo Innaca panaca & Inacapanaca & Iñaca panaca & Inaca panaca/ Hatun ayllo & Atun ayllu \\
\hline Capac ayllo & Capacayllu & Capac ayllo & Capac ayllo & Capac ayllu \\
\hline
\end{tabular}

FIG. 1 - Tableau comparatif de la nomenclature des groupes aristocratiques selon diverses sources.

L'ethnohistoire andiniste a consacré l'usage du terme panaca pour désigner les lignages royaux de Cuzco. Il apparaît cependant qu'hormis chez Sarmiento de Gamboa, ce vocable, auquel vient parfois s'ajouter le terme ayllu, ne se trouve associé dans les sources qu'à un nombre restreint de groupes, les autres se voyant simplement désignés comme ayllu ou ne recevant pas de qualificatif. Du fait que les auteurs n'ont pas commenté ce phénomène, mais aussi parce que le modèle classique de formation des groupes aristocratiques conduisait à postuler leur homogénéité ${ }^{3}$, ces variations ont pu être considérées comme un aléa de la rédaction. On observe pourtant qu'à deux exceptions près, les quatre sources présentent une information identique : Chima, Raura(hua), Sucsu et Iñaca (quand il est mentionné) se voient systématiquement associer le terme panaca, tandis que Hahuaynin, Uscamayta et Apomayta ne sont pas qualifiés ou se voient désignés comme ayllu, à la manière de Hatun et Capac. Seul le traitement de Vicaquirau et Aucaylli marque un flottement, et il n'existe, dans les deux cas, qu'une seule source divergente. Le contexte dans lequel Las Casas évoque 
Vicaquirau pourrait d'ailleurs expliquer qu'il soit le seul à lui associer le vocable panaca : énumérant les groupes suivant un ordre hiérarchique descendant où Vicaquirau se trouve mentionné à la suite de trois panaca (Iñaca, Sucsu et Aucaylli ${ }^{4}$ ), l'application du qualificatif panaca pourrait alors se comprendre par un effet d'entraînement mécanique. Molina, dont la liste recoupe les autres sources, s'en démarque cependant pour Aucaylli, qualifié d'ayllo, écart dont il faut peut-être chercher la raison dans l'assonance de «Aucaylli » qui semble appeler « ayllu ». Discordances qui s'expliquent d'autant mieux qu'hormis la fidélité au matériel fourni par leurs informateurs, les auteurs ne pouvaient prendre en considération des différences terminologiques qui ne leur auraient pas été expliquées et devaient dès lors leur sembler arbitraires ${ }^{5}$.

Si l'on ne peut exclure définitivement la possibilité d'emprunts entre ces différentes sources dans le détail de la terminologie qu'elles proposent, cette éventualité apparaît pourtant peu vraisemblable. En effet, les variations dans la transcription des noms indigènes ou le contenu de l'information lui-même - notamment dans le cas de Iñaca/Hatun - témoignent en faveur de l'indépendance des sources, sans compter que la plupart d'entre elles résultaient d'enquêtes de terrain réalisées auprès de membres de l'aristocratie cuzquénienne, contexte dans lequel on peut estimer que le nom des groupes sociaux constituait une information aisément accessible.

En recoupant l'information provenant des différentes sources, suivant la transcription conventionnelle, on obtient le tableau suivant (Figure 2).

\begin{tabular}{cll}
\multirow{2}{*}{ Urin Cuzco } & Souverains successifs & Groupe correspondant \\
& Manco Capac & Chima panaca (ayllu) \\
& Sinchi Roca & Raura(ua) panaca (ayllu) \\
& Lloque Yupanqui & Hahuaynin (ayllu) \\
\multirow{3}{*}{ Hanan Cuzco } & Mayta Capac & Uscamayta (ayllu) \\
& Capac Yupanqui & Apomayta (ayllu) \\
& Yahuar Hoca & Vicaquirau (ayllu) \\
& Viracocha Inca & Aucaylli panaca $($ ayllu) \\
& Pachacuti Inca Yupanqui & Sucsu panaca (ayllu) \\
& Topa Inca Yupanqui & Iñaca panaca/Hatun ayllu
\end{tabular}

FIG. 2 - Les souverains incas et leurs groupes de descendants supposés.

\section{Apports documentaires complémentaires}

Deux documents produits collectivement par l'ensemble de l'élite cuzquénienne viennent appuyer les données des sources classiques. Au dernier chapitre de ses Comentarios, Garcilaso fait état d'une lettre de 1603 par laquelle les représentants de la noblesse de Cuzco lui demandaient, ainsi qu'à Melchor 
Carlos et Alonso de Mesa, de les représenter auprès de la couronne pour obtenir les privilèges fiscaux dus à leur rang. Dans ce document figurait un portrait de chaque souverain, associé au nom du groupe de ses descendants dont l'effectif était également précisé. Le chroniqueur en rapporte le contenu au style indirect :

A la descendencia de Manco Capac llaman Chima Panaca : son cuarenta incas los que hay de aquella sucesión. A la de Sinchi Roca llaman Raurava Panaca : son sesenta y cuatro incas. A la de Lloque Yupanqui, tercero Inca, llaman Hahuanina Aillu : son sesenta y tres. A los de Capac Yupanqui llaman Apu Maita : son cincuenta seis. A los de Maita Capac, Quinto Rey, llaman Usca Maita : son treinta y cinco. A los de Inca Roca dicen Uicaquirau : son cincuenta. A los de Yahuar Huacac, séptimo Rey, llaman Ailli [sic] Panaca : son cincuenta y nueve. A los de Viracocha Inca dicen Zoczo Panaca : son sesenta y nueve. A la descendencia del Inca Pachacutec y a la de su hijo, Inca Yupanqui, juntándolas ambas, llaman Inca [sic] Panaca [...] A la descendencia de Tupac Inca Yupanqui llaman Capac Aillu. (Garcilaso de la Vega 2003 [1609], p. 730 [souligné par l'auteur, L. S.])

Si l'on observe quelques distorsions dans l'ordre de succession des souverains et la transcription des noms, en revanche la distribution du vocable panaca concorde parfaitement avec les sources déjà mentionnées.

Un document d'archives plus ancien présente une information similaire. En 1577, Gabriel Paniagua de Loayza, alors corregidor de Cuzco, enfreint une coutume bien établie en contraignant à la corvée des membres de l'aristocratie inca que leur statut préservait jusqu'alors. Deux ans plus tard, sous la direction de Felipe Saire Topa, " alcalde mayor de los naturales » et fils de Paullu, plus de trente nobles cuzquéniens entament une procédure pour faire valoir leur droit à être exemptés de corvée. Des personnages importants sont appelés à témoigner, parmi lesquels Manso Sierra de Leguizamo et Alonso de Mesa (père de l'homonyme précédemment cité), deux vieux conquistadores, ainsi que Damián de la Bandera et Cristóbal de Molina. La liste des plaignants figurait sur un feuillet manquant au document. Le fait qu'ils se présentent collectivement à la fois comme " yngas descendientes de Mango Capac », ancêtre tutélaire de l'ensemble de la noblesse cuzquénienne ${ }^{6}$, et comme "yngas descendientes de los yngas señores que fueron destos reynos ", laisse cependant supposer que la plupart des groupes était représentée, expliquant ainsi la teneur de la première question composée par les demandeurs et adressée aux témoins :

Primeramente si conocen a los yngas desendientes de los señores deste reino questan poblados en las perroquias desta ciudad del Cuzco que son onze ayllos llamados chima panaca, rauraua panaca, hahuaynin ayllo $y$ apomayta $y$ uscamayta $y$ vicaquerao $y$ aucaylle panaca $y$ cucco panaca $e$ ynaca panaca $y$ capac ayllo $y$ tomebamba contenidos en la memoria que se muestre a los testigos. ([1579] AGI, Lima 472, cuad. 7, f. 2 [souligné par l'auteur, L. S.])

Le document enregistre onze groupes aristocratiques, « Tomebamba » venant s'ajouter à la liste standard ${ }^{7}$. On constate cependant que, s'agissant de la distri- 
bution du terme panaca, le document reproduit exactement l'information des chroniques et le contenu de la lettre rapportée par Garcilaso de la Vega. Comme chez ce dernier, on note également l'absence du qualificatif ayllu après le terme panaca comme d'ailleurs après le nom de la plupart des groupes désignés comme « ayllus » dans les autres sources. Incidemment, au regard de l'ensemble des autres sources, ce constat pourrait conduire à privilégier comme forme adéquate " panaca ayllu » plutôt que " panaca ». Ce qui inciterait peut-être à voir dans le terme panaca le marqueur potentiel d'un type spécifique de groupe social ou de parenté (ayllu), plutôt que l'appellation générique de ces groupes eux-mêmes. Les sources offrant des indices mais aucune certitude à ce sujet, la prudence impose toutefois une notation qui admette les deux possibilités : "panaca (ayllu) ", notamment lorsqu'il s'agit d'opposer ces groupes à ceux simplement désignés comme ayllu (Figure 3).

\begin{tabular}{|c|c|c|c|}
\hline \multirow{6}{*}{ Urin Cuzco } & Sources (1559-1575) & Garcilaso (1603) & Probanza de 1579 \\
\hline & Chima panaca (ayllu) & Chima panaca & Chima panaca \\
\hline & Raura(hua) panaca (ayllu) & Rauraua panaca & Rauraua panaca \\
\hline & Hahuaynin (ayllu) & Hahuanina aillu & Hahuaynin ayllo \\
\hline & Uscamayta (ayllu) & Usca Maita & Uscamayta \\
\hline & Apomayta (ayllu) & Apu Maita & Apomayta \\
\hline \multirow[t]{5}{*}{ Hanan Cuzco } & Vicaquirau (ayllu) & Uicaquirau & Vicaquirau \\
\hline & Aucaylli panaca (ayllu) & Aillipanaca & Aucayllepanaca \\
\hline & Sucsu panaca (ayllu) & Zoczo panaca & Cucço panaca \\
\hline & Iñaca panaca/Hatun ayllu & Inca panaca & Ynaca panaca \\
\hline & Capac ayllu & Capac aillu & Capac ayllo \\
\hline
\end{tabular}

FIG. 3 - Tableau synthétique des sources citées.

\section{Une illustration : les cérémonies de la Situa}

Sans toutefois permettre d'éclairer le sens de la distinction entre ayllu et panaca (ayllu), certaines données semblent au moins témoigner de son effectivité pratique dans la vie sociale et rituelle cuzquénienne. À l'occasion des premières pluies du mois de septembre qui favorisaient l'apparition de maladies, les cuzquéniens célébraient les cérémonies de la Situa ${ }^{8}$, marquées notamment par un rite de purification mobilisant l'ensemble des groupes sociaux. Depuis la place centrale, quatre cents « guerriers » partaient simultanément par groupes de cent en direction des quatre $s u y u$, repoussant le mal jusqu'aux limites du territoire de la cité. Molina indique la direction prise à cette occasion par les dix groupes aristocratiques (Molina 1989, p. 75 [erreurs de transcription manifestes rectifiées par l'auteur]) (Figure 4). 


$\begin{array}{ll}\text { Chinchasuyu (nord-ouest) } & \text { "Capac ayllu y Atun ayllu y Vicaquirao } ~ \\ \text { Antisuyu (nord-est) } & \text { "Cuscupanaca ayllo, Aucaylli ayllo } ~ \\ \text { Collasuyu (sud-est) } & \text { "Uscamayta ayllo y Apomayta ayllu y Hahuaynin ayllu } ~ \\ \text { Cuntisuyu (sud-ouest) } & \text { "Raurapanaca aillu y Chimapanaca aillu } ~\end{array}$

FIG. 4 - Distribution géographique des groupes aristocratiques lors de la Situa.

Si l'organisation spatiale du rite exprime clairement le dualisme de la société cuzquénienne - les groupes de Hanan Cuzco se dirigeant vers Chinchaysuyu et Antisuyu (moitié Hanan du territoire), ceux d'Urin Cuzco vers Cuntisuyu et Collasuyu (moitié Urin) -, elle semble également mettre en scène une autre partition. Dans chacune des moitiés Hanan et Urin, le quart supérieur (Chinchaysuyu, Collasuyu) se trouve en effet réservé aux ayllu, et le quart inférieur (Antisuyu, Cuntisuyu) aux groupes désignés comme " panaca ayllu ». Opérant à la manière d'une grille classificatoire exprimant à la fois la hiérarchie des moitiés et la partition qui nous occupe, il semble toutefois que l'espace mobilisé par les groupes sociaux ne se limitait pas au cadre temporel du rite. On observe en effet un dispositif similaire dans la distribution des ceque attribués aux groupes aristocratiques (Cobo 1956, pp. 169-186), dont tout indique qu'ils devaient déterminer en amont l'espace rituel de la Situa ${ }^{9}$. Il faudrait dès lors envisager que la distinction entre ayllu et panaca (ayllu) ait pu, au même titre que le dualisme Hanan Cuzco/Urin Cuzco, conditionner l'attribution de certains ceque. Il convient de signaler à ce propos un fait notable, abondamment attesté par la documentation d'archives ${ }^{10}$ : contrairement à une hypothèse répandue ${ }^{11}$, la distribution des ceque n'était pas corrélée à l'implantation territoriale des groupes qui les avaient à charge, du moins si l'on considère la répartition des terres antérieure aux réformes des années 1560-1570. Ce constat ne saurait évidemment constituer en soi un argument déterminant en faveur du rôle de la dichotomie ayllu/panaca (ayllu) dans la distribution des ceque. Il suggère néanmoins que l'attribution des ceque " aristocratiques » dépendait certainement moins de facteurs matériels liés à l'espace physique en tant que tel que de schèmes et de représentations qui s'y trouvaient projetés, parmi lesquels la distinction entre ayllu et panaca (ayllu) avait apparemment sa part (Figure 5).

Dans son approche du système de parenté inca, Zuidema s'était attaché à différencier les concepts d'ayllu et de panaca, une distinction terminologique qui, pour lui, dépendait essentiellement du point de vue du locuteur. Interprétant panaca comme un dérivé de pana (" sœur d’un homme »), ce vocable désignerait le groupe frères-sœurs ( « sœurs » qualifiant également les cousines croisées patrilatérales jusqu'au quatrième degré), considéré du point de vue masculin, suivant une logique de "parentèle d'orientation ». Le terme ayllu qualifierait le même 


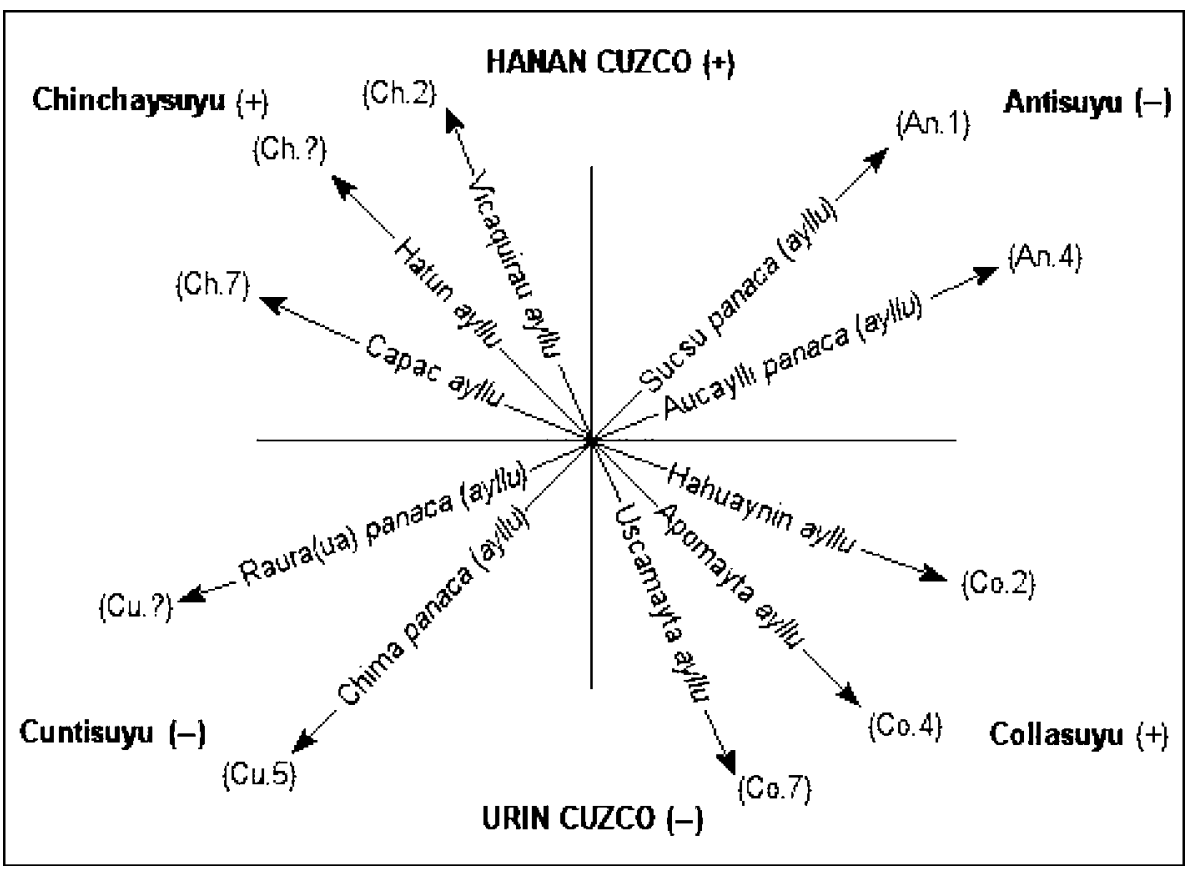

FIG. 5 - Ayllu et panaca (ayllu) dans l'espace de la Situa et le réseau des ceque*.

* L'auteur utilise ici le mode de notation conventionnel des ceque, en usant des abréviations admises pour les suyu $(\mathrm{Ch} .=$ Chinchaysuyu, An. = Antisuyu, Co. = Collasuyu, Cu. = Cuntisuyu). Par exemple : An. $1=1^{\mathrm{er}}$ ceque d'Antisuyu.

groupe, mais du point de vue de l'ancêtre masculin, se référant ainsi à une " parentèle de procréation » (Zuidema 1977, p. 256). Admettant par ailleurs l'existence de sections exogames matrilinéaires à l'intérieur du groupe de descendants d'un même ancêtre, et la possibilité pour un homme de contracter un mariage endogame ou exogame, il suggérait également que, selon qu'il se mariait à l'intérieur ou à l'extérieur de son groupe d'appartenance, cet homme pouvait désigner celui-ci comme panaca (matrilinéaire) ou ayllu (patrilinéaire) (Zuidema 1995 [1964], pp. 29, 73-74 et 299). Considérant cependant - notamment sous l'influence de la liste fournie par Sarmiento de Gamboa - que seul Capac était invariablement désigné comme ayllu quand les neuf autres groupes pouvaient être qualifiés de panaca, il voyait dans ce phénomène le reflet du point de vue des souverains successifs (selon lui tous membres de Capac ayllu) sur l'organisation sociale de Cuzco (ibid., pp. 34-35). Néanmoins l'analyse attentive des sources montre que, contrairement à ce qu'envisageait Zuidema, le terme panaca n'était associé qu'à un nombre limité de groupes aristocratiques. Par ailleurs, elle 
témoigne également de la stabilité d'une terminologie différenciée, difficilement réductible à une question de point de vue, cela même à considérer la double dénomination problématique de Hatun ayllu/Iñaca panaca (ayllu). Les cérémonies de la Situa et, au-delà, la distribution spatiale des ceque attribués aux différents groupes suggèrent au contraire la validité et l'importance de cette distinction terminologique (ou de la réalité qu'elle recouvre) pour la société cuzquénienne elle-même.

Les travaux récents de Zuidema témoignent d'une évolution quant à sa conception des panaca, désormais moins envisagées comme des groupes de parenté ou des classes matrimoniales que comme des entitées composites chargées de l'administration des divisions territoriales de la vallée (chapa), mais aussi investies de fonctions calendaires, et fonctionnant comme des classes d'âge (prenant successivement en charge les membres de la noblesse cuzquénienne), dont chaque souverain aurait confié le commandement à des fils issus de mariages polygames (Zuidema 2010, chap. 5 et 7). Sa réinterprétation du concept conduit ainsi Zuidema à reconnaître la qualité de panaca à l'ensemble des groupes aristocratiques cuzquéniens, y compris aux ayllu Capac et Hatun (ibid., par exemple pp. 373, 406 et 421).

\section{Panaca}

Son usage restreint - et problématique, si l'on admet la forme panaca aylluvenant contester une fonction supposée de dénominateur standard des groupes aristocratiques cuzquéniens, le terme panaca pose un problème de définition que les rares données disponibles ne permettent pas de résoudre de manière satisfaisante. Si la seule occurrence dans les lexiques coloniaux présente peu d'utilité ${ }^{12}$, la solution proposée par Zuidema - après Valcárcel (1925, p. 38) - à partir de pana (« sœur d'un homme ») ne semble pas avoir été commentée par les quechuistes. Quant à l'assertion de Sarmiento de Gamboa selon lequel «panaca quiere decir descender » (2001, p. 64), elle apparaît dans son contexte - l'évocation du lignage de Manco Capac - comme une explication ad hoc visiblement dépourvue de fondement linguistique.

En réalité, hormis son usage par l'aristocratie cuzquénienne, il n'existe, dans le corpus des sources connues, que deux utilisations du terme. La première chez Polo de Ondegardo qui, évoquant dans sa Relación manuscrite de 1571 les « maisons des femmes choisies » (acllahuasi), précise que chacune disposait d'un " gobernador [...] cuyo nombre Apopanaca, que significa este género de oficio » (Polo de Ondegardo 1990 [1571], p. 79), également chargé de choisir les jeunes filles « de buena disposición y gesto de ocho años para abajo ». S'appuyant sur Polo de Ondegardo, Acosta écrira que « cada monasterio tenía su vicario o gobernador, llamado Apo panaca » (Acosta 1954 [1590], p. 156). La seconde mention figure chez Pérez Bocanegra, qui l'utilise à plusieurs reprises dans son Ritual formulario 
(1631) sous la forme « apu panaca », afin de traduire en quechua la fonction du pape Paul V ${ }^{13}$, "vicaire du Christ » $(a p u=$ seigneur $)$, semblant rejoindre ainsi l'interprétation suggérée par Acosta à partir du texte de Polo. On observe cependant que Pérez Bocanegra emploie par ailleurs à deux reprises panaca comme un équivalent d'ayllu, sans toutefois identifier les deux termes ${ }^{14}$. Dès lors, à moins d'admettre un double sens ou une homophonie, il faudrait envisager que Pérez Bocanegra, pourtant quechuiste expérimenté, ait pu emprunter directement cet usage au contexte cuzquénien, sans que rien ne puisse garantir qu'il n'ait pas fait de même avec un « apu panaca » qu'il aurait pu tirer d'Acosta, directement ou non. Le succès de l'Historia natural y moral du père jésuite vient conforter cette hypothèse, renforcée par la postérité littéraire d'un Apo panaca " vicaire », repris tel quel chez d'autres auteurs du XVII ${ }^{\mathrm{e}}$ siècle, comme dans le $D e$ sacrorum immunitatibus (1591) d'Anastasio Germonio, réédité dans ses Ópera omnia (1623) huit ans avant la publication du Ritual formulario de Pérez Bocanegra, et où le parallélisme romain et l'évocation «pontificale » - quoique se rapportant en l'occurrence à la Rome païenne - sont déjà à l'œuvre : "Scribit Josephus Acosta [...] Capiebantur eiusmodi puellae annos circiter octo natae, non, ut Vestales Romae, a Pontifice Maximo, sed ab eius Vicario, cuio nomen erat Apopanaca. 》 (Germonius 1623, p. 23).

Au final, le texte originel de Polo de Ondegardo demeure donc la seule source réellement fiable pour un terme dont il propose une interprétation, mais sans fournir une traduction à proprement parler. Du reste, il pourrait apparaître surprenant que le système administratif incaïque, complexe et fortement charpenté, ait réservé le titre de " gouverneur/vicaire » aux seuls fonctionnaires des acllahuasi.

En admettant pourtant que le vocable panaca désigne effectivement une fonction de représentation administrative ou politique, l'incertitude quant à la forme adéquate (panaca ou panaca ayllu) serait définitivement résolue en faveur de la seconde, et l'on serait conduit à traduire par exemple «Chima panaca ayllu » par « l'ayllu du gouverneur/vicaire Chima ». Envisageable pour Chima, Raura, Sucsu et Aucaylli - quoiqu'il resterait alors à expliquer pourquoi les noms des groupes se référeraient à des personnages autres que leurs ancêtres tutélaires -, l'hypothèse apparaît plus difficilement tenable pour Iñaca qui ne constitue pas un nom propre, mais désigne une catégorie spécifique de femmes de l'aristocratie cuzquénienne ${ }^{15}$. L'interprétation de panaca comme fonction personnelle ne pourrait alors se maintenir qu'en supposant une forme génitive (« gouverneur/ vicaire $d e(s) »)$, qui appliquée à Chima, Raura, Aucaylli et Sucsu placerait toutefois le référent identitaire de l'ayllu hors du groupe lui-même, qui dès lors ne descendrait plus d'hypothétiques Chima ou Sucsu mais de leurs « gouverneurs/ vicaires » anonymes.

La nette prédominance de la notation «panaca » dans les sources classiques et la documentation d'archives en a éclipsé une autre, minoritaire mais ancienne, attestée à la fois chez le dominicain quechuiste Santo Tomás et dans la Relación de 
las huacas : « pañaca ». Au chapitre XVIII de sa Grammática (1560, env. 1550 pour le manuscrit) portant sur les patronymes, Santo Tomás définit les "ingas » comme l'ensemble des descendants de " Mango Inga », précisant que ce vaste "linage tiene entre ellos otros particulares nombres y linages: que el principal se llama capac ayllo, otro igñaca pañaca ayllo, otro çucco pañaca ayllo [souligné par l'auteur, L. S.], y assí muchos otros » (Santo Tomás 1995 [1560], p. 118). Quant à la Relación de las huacas (1559-1561), si elle use de la notation majoritaire pour transcrire " Chima panaca », " Aucailli panaca » et " Inaca panaca », elle enregistre également «Cubçu pañaca ayllu » (Cobo 1956, p. 175), suivant une formulation [pañaca + ayllu] similaire à celle proposée par Santo Tomás. La question nécessiterait évidemment une analyse philologique appropriée ; toutefois quelques observations sommaires restent possibles. En regard des deux notations " panaca»/« pañaca», on constate par exemple que les transcriptions « inaca »/《 iñaca » présentent le même rapport quant à leur fréquence d'usage, les sources originales usant majoritairement de la première forme. Ceci à l'exception notable de Las Casas, qui suit en cela Santo Tomás, sa source potentielle, présentant en l'occurrence une notation (igñaca) plus conforme à la réalité. Sans être proprement déterminant, car susceptible d'interprétations multiples, un autre constat pose néanmoins question : en admettant la validité probable de la notation majoritaire, on peut être interpelé par le fait qu'en évoquant son « Apo panaca ", traité lapidairement, Polo de Ondegardo, pourtant excellent connaisseur du terrain cuzquénien, ne laisse pas entrevoir la conscience d'une relation possible avec la terminologie des groupes sociaux de Cuzco. Il convient enfin de signaler que si paña existe en quechua (" Paña. Mano derecha. Paña chiru, o paña huacta. El lado derecho », González Holguín 1989 [1608], p. 277), la forme pañaca est également attestée, de manière contemporaine et dans des régions éloignées de Cuzco, où elle désigne l'institution andine du "mariage probatoire » : pañaca servinakuy ou sirvicia (Huánuco), enregistré à Ancash sous la forme « mushiapanaki » (Rubio Correa 1985, p. 67). Si donc certains faits empêchent d'écarter totalement l'hypothèse " pañaca », ils ne sont toutefois pas suffisamment consistants pour renoncer à « panaca » comme forme de référence, dont, en l'état, une définition claire s'avère malgré tout difficilement accessible. Il reste que dans les rares contextes bien définis où il apparaît, le terme se trouve associé à des institutions féminines : les acllahuasi, "maisons des femmes choisies », et la classe des femmes iñaca. Ce qui, sous réserve de sa validité sur le plan linguistique, cela pourrait aller dans le sens de l'hypothèse d'une racine pana (« sœur d'un homme »), suffixée en -ka ou en -qa (voir par exemple Hernández Astete 2008, pp. 41-42).

L'analyse de la nomenclature des " panaca » pourrait peut-être offrir un moyen d'approche complémentaire si elle ne se heurtait également à divers obstacles, notamment philologiques. Chima ne figurant pas dans les lexiques quechua coloniaux, Cerrón Palomino (1998, p. 421) suggère une étymologie 
aymara qui le ferait dériver de chima (kha), " [...] morado que tira a encarnado » (Bertonio 1984 [1612], $2^{\mathrm{e}}$ partie, p. 85). S'agissant de raura ou raurahua, la principale difficulté réside dans l'incertitude entre les deux dénominations du groupe, pouvant relever respectivement du quechua raura, «ardent», « enflammé » (González Holguín 1989, pp. 314-315) et de l'aymara laurahua, « plumes de l'oiseau caque » (Cerrón Palomino, ibid.; Bertonio ibid., p. 192). Quant à aucaylli - peut-être dérivé d'aucay haylli, " chant triomphal et fêtes de victoires » (González Holguín ibid., p. 38) - et sucsu, « faible, épuisé, amaigri » (ibid., p. 331), leur interprétation nous semble dépendre de référentiels trop mal connus pour formuler des hypothèses solides. Pour autant, en considérant la validité probable de la forme " panaca ayllu », la dimension qualitative de cette nomenclature permet d'envisager que chima, raura, sucsu... puissent non pas constituer les noms propres de groupes relevant d'une catégorie de groupes sociaux dénommés panaca, différenciés des ayllu, mais désigner plutôt différents segments ou manifestations d'une même institution sociale (féminine ?) spécifique, qui trouverait peut-être un parallèle ou un équivalent dans les acllahuasi, encore mal connus. Dans ce cas, par exemple, "Raura panaca ayllu » pourrait s'interpréter comme "l'ayllu de la panaca ardente », "Sucsu panaca ayllu », " ayllu de la panaca chétive » ou encore " Iñaca panaca ayllu », " ayllu de la panaca de l'iñaca (= des femmes iñaca)».

Sans être plus aisément interprétable, la nomenclature des ayllu contraste néanmoins avec celle des panaca en ce qu'elle laisserait entrevoir plus nettement une dimension classificatoire. Pour les ayllu d'Urin Cuzco, celle-ci s'exprime non seulement dans l'emploi d'un terme de parenté : hahuaynin, dérivé de hahua(y), « petit-fils » (ibid., p. 145), mais aussi dans l'opposition entre apu « seigneur » (ibid., p. 31) et usca « mendiant» (ibid., p. 358), associés au terme mayta, dont la signification reste à déterminer. Pour Hanan Cuzco, à côté de vicaquirau, « berceau (quirau) d'excréments (vecca) » (ibid., pp. 310, 350), hatun: " grand » et capac: "royal, riche" dénoteraient également un aspect classificatoire, déjà relevé par Zuidema (1986, p. 54, 1995 [1964], pp. 214-215). Si des éléments suffisamment probants font défaut pour éclairer le concept de panaca, en revanche la présence, dans chaque moitié, de trois ayllu apparemment dotés d'une nomenclature classificatoire permet de faire appel à un modèle bien attesté, peut-être susceptible de ramener un pan de la réalité cuzquénienne à des structures andines connues.

\section{Les trois ayllu de Hanan Cuzco et la tripartition collana, payan et CAYAU}

Le terme ayllu désigne à la fois un lignage, un groupe de parenté, et une arme $^{16}$ de type bolas, constituée de trois lanières jointes lestées à leur extrémité ${ }^{17}$. L'affinité des deux référents est aussi structurelle : comme l'arme, 
le groupe social apparaît couramment subdivisé en trois sections souvent désignées par les termes Collana, Payan, Cayau. Susceptible d'exprimer différentes réalités (degrés de parenté, rangs généalogiques ou hiérarchiques...) en fonction de la nature des trois sections, la tripartition des ayllu peut également opérer à plus vaste échelle, les tripartitions s'emboîtant alors à la manière des poupées gigognes suivant une configuration attestée au XVI ${ }^{\mathrm{e}}$ siècle dans différentes zones des Andes centrales (Kirchhoff 1949, p. 303 ; Zuidema 1995 [1964], p. 97). Or certaines données cuzquéniennes invitent à interroger la tripartition collana, payan, cayau comme cadre d'analyse possible des trois ayllu des deux moitiés, en particulier ceux de Hanan Cuzco. En effet, comme le montre un document publié par Rowe, cette tripartition s'appliquait déjà à la structure de Capac ayllu. Surtout, l'organisation interne de ce groupe soulève une série de problèmes qui ne semblent pouvoir se résoudre qu'à la condition d'envisager Capac ayllu lui-même comme la section d'une structure plus large.

\section{L'organisation interne de Capac ayllu}

Dans les documents relatifs à une procédure juridique conduite en 1569 par ses représentants, l'ayllu Capac apparaît divisé en trois sections (Figure 6) : Collana, Payan, et Cayao (Rowe 2003c, pp. 79-116). L'ayllu Collana est représenté par neuf personnes se proclamant "nietos de Topa Ynga Yupangui », successeur de Pachacuti Inca Yupanqui, l'ayllu Payan par huit «nietos de Amaro Topa Ynga, hermano de Topa Ynga Yupangui », et l'ayllu Cayao par cinq « nietos de Topa Yupangui ynga hermano de Topa Ynga Yupangui señor que fue deste reyno " (ibid., p. 100).

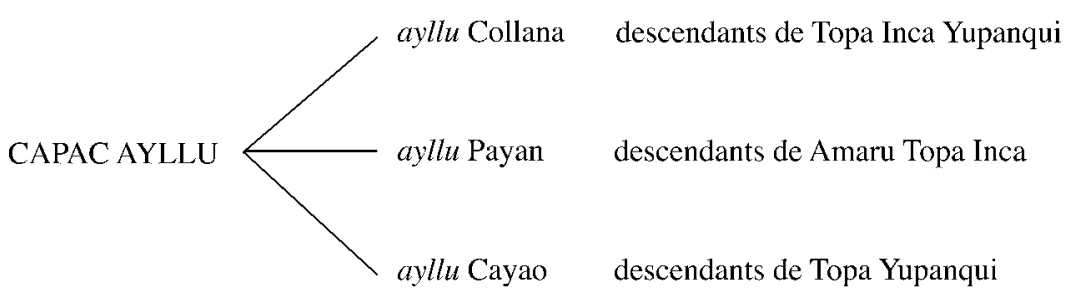

FIG. 6 - La structure de Capac ayllu d'après la probanza de 1569.

Rowe qualifiait la structure de Capac ayllu d'《 anormale » (ibid., p. 81), Pärssinen la trouvant quant à lui « très surprenante » (Pärssinen 2003, p. 192). De fait, elle venait heurter la théorie classique de constitution des groupes 
aristocratiques, exigeant que Capac ayllu regroupe les seuls descendants de l'Inca Topa Inca Yupanqui, fils et successeur de Pachacuti Inca Yupanqui. Or, contre toute attente, l'ayllu rassemblait également les descendants des collatéraux du souverain, que l'on s'attendrait à retrouver dans Hatun ayllu, considéré comme le lignage propre de Pachacuti Inca Yupanqui. Pour expliquer cette « anomalie », Rowe faisait appel à Las Casas : selon ce dernier, en compensation de son éviction de la succession au trône en faveur de Topa Inca Yupanqui, Amaru Topa Inca (ancêtre de l'ayllu Payan) aurait été nommé « cabeza y capitán del principal linaje real, llamado Capac ayllo » (cité in Rowe 2003c), ce qui expliquerait l'affiliation de ses descendants à Capac ayllu plutôt qu'à Hatun ayllu. Pour autant, comme le concédait Rowe, rien ne justifiait la présence au sein de Capac ayllu des descendants de Topa Yupanqui, ancêtre de l'ayllu Cayao (ibid., p. 82). Tentant également d'expliquer la structure paradoxale de l'ayllu, Pärssinen proposait une théorie triarchique du pouvoir inca qui aurait vu Topa Inca Yupanqui régner avec ses deux frères. Capac ayllu aurait alors rassemblé les descendants des trois Incas co-régnants, inaugurant une structure socio-politique inédite censée résulter d'une mutation historique pour laquelle Pärssinen ne propose cependant pas d'explication (Pärssinen 2003, pp. 192-193). Confronté au même problème, Zuidema voyait plutôt dans la tripartition l'effet d'une représentation théorique étrangère à la structure réelle de Capac ayllu (Zuidema 1995, p. 64). La documentation d'archives confirme pourtant les assertions des membres de l'ayllu, notamment quant à l'existence d'une section rassemblant les descendants de Amaru Topa Inca, attestée dès $1561^{18}$.

\section{Capac ayllu, HatunlIñaca et Pachacuti Inca Yupanqui}

Les propositions de Rowe et Pärssinen pour expliquer la structure paradoxale de Capac ayllu reposaient sur un double postulat: d'une part, l'effectivité du modèle classique de constitution des lignages royaux, d'autre part, le fait que Capac ayllu devait être considéré comme le groupe de descendants de Topa Inca Yupanqui. La théorie de formation des groupes aristocratiques supposant entre eux une stricte homogénéité contredite par la dichotomie ayllu/panaca (ayllu), il convient alors de s'attacher aux données factuelles. Or, si l'on ne peut nier l'appartenance des descendants de Topa Inca Yupanqui à Capac ayllu, il est également incontestable que, si l'on y retrouvait en outre les descendants de deux autres fils de Pachacuti Inca Yupanqui, c'est qu'il s'agissait de fait d'un groupe de descendants de Pachacuti Inca Yupanqui lui-même. Sur ce point, le témoignage de Betanzos, marié à une descendante du souverain, est sans ambigüité : " A los descendientes deste Inca Yupanqui llamavan desde entonces hasta hoy Capac aillo Ynga Yupanqui Haguaymin que dice de linaje de reyes y descendientes y nietos de Ynga Yupangue [= Pachacuti Inca Yupanqui], y estos son los mas sublimados y tenidos en más entre los del Cuzco »(Betanzos 1987 [1551], p. 150). Une 
assertion répétée plus loin : "Capac Aillo que ellos dicen que era el linaje de Ynga Yupangue " (ibid., p. 194).

S'agissant dès lors d'expliquer l'existence d'un autre groupe de descendants de Pachacuti, Hatun ayllu ou Iñaca panaca, les informations recueillies par Sarmiento de Gamboa sur la descendance du souverain fournissent un premier élément de réponse : " [Pachacuti Inca Yupanqui] tuvo cuatro hijos legítimos en su mujer Mama Anahuarqui, tuvo cien hijos varones y cincuenta hijas bastardas a los cuales por ser muchos llamó Hatun ayllu, que quiere decir "gran linaje". Llámase por otro nombre este linaje Inaca panaca ayllu. » (Sarmiento de Gamboa 2001, p. 127).

Mais la formulation du chroniqueur peut prêter à confusion. Une première lecture pourrait en effet conduire à associer les quatre " hijos legitimos" aux " cien hijos varones » et « cincuenta hijas bastardas » regroupés dans Hatun ayllu. L'identification des «fils légitimes" de Pachacuti et Mama Anahuarqui ne présente pas de difficulté : il s'agit bien de Topa Inca Yupanqui et de ses frères Amaro Topa Inca et Topa Yupanqui, revendiqués comme ancêtres par les membres de Capac ayllu, à ceci près que le chroniqueur dénombre quatre « fils légitimes » au lieu de trois ${ }^{19}$. Malgré son imprécision, la formule de Sarmiento de Gamboa permettrait donc de distinguer deux groupes de descendants de Pachacuti : d'un côté, des fils « légitimes » fondateurs de Capac ayllu, de l'autre, une vaste progéniture issue de femmes secondaires qui aurait composé Hatun ayllu/Iñaca panaca (ayllu). Une distinction entre fils primaires et secondaires qui recoupe parfaitement le champ d'application d'une classification collana-payan (Zuidema 1989, p. 93) et qui pourrait d'ailleurs s'exprimer dans les noms mêmes des deux groupes. Bien sûr, comme le suggère Sarmiento de Gamboa, le nom de « Hatun ayllu» (« grand ayllu») pourrait se rapporter au grand nombre des enfants «bâtards» de Pachacuti, tandis que «Capac ayllu» (« ayllu royal/ riche/puissant ») semblerait approprié pour qualifier le groupe rassemblant les fils du souverain et de sa Coya, ainsi que les descendants du successeur au trône. Toutefois, la documentation historique et ethnographique incite à questionner cette interprétation commode de la nomenclature, certaines informations témoignant également de l'usage de capac et hatun comme synonymes de collana et payan dans certaines structures tripartites, comme à Anta ou Curahuasi (Zuidema 1995 [1964], pp. 214-215).

Au final, la composition des deux ayllu cuzquéniens inviterait à rechercher un hypothétique groupe en position cayau, susceptible de compléter la tripartition de manière adéquate, c'est-à-dire suivant une logique hiérarchique descendante ou une classification de parenté exprimant peu ou prou une forme d'infériorité statutaire par rapport aux membres de Capac et Hatun. De toute évidence, Vicaquirau, en tant que troisième ayllu de Hanan Cuzco, s'imposerait alors comme le candidat le plus vraisemblable. 


\section{L'ayllu Vicaquirau comme section Cayau?}

À partir des années 1560, la majorité des chroniqueurs reconnaissait dans l'ayllu Vicaquirau le lignage d'Inca Roca, premier souverain de Hanan Cuzco. Cependant, Betanzos en proposait en 1551 une toute autre définition, assimilant le groupe à une catégorie particulière de fils secondaires de l'Inca, issus d'unions avec des femmes « étrangères » ou d'extraction non aristocratique, et désignés comme huaccha concha:

eran hijos bastardos de señores aunque eran de su linaje los cuales habian habido en mujeres extrañas de su nación e de baja suerte a los cuales hijos ansí habidos llaman ellos Guacchaconcha que quiere decir deudos de pobre gente e baja generación y estos tales aunque sean hijos del Ynga son llamados ansí e no son tenidos ni acatados ninguno destos ansí hombres como mujeres de los demás señores si no como por un orejón de los otros comunes (Betanzos 1987, pp. 77-78)

Tandis que concha désigne l'enfant de la sœur d'un homme ${ }^{20}$, huaccha qualifie aussi bien le «pauvre» que l'« orphelin » ${ }^{21}$. Si l'interprétation de huaccha concha comme « neveu pauvre » (Zuidema 1989, p. 99) peut répondre à une infériorité statutaire soulignée par Betanzos, la traduction « neveu orphelin » semble également suggestive : en désignant une partie de sa progéniture comme « enfants de ses sœurs », l'Inca pourrait alors se positionner comme l'oncle maternel d'individus qui de ce fait n'auraient pas de père. L'oncle maternel (caca) appartenant, dans le cadre d'une filiation patrilinéaire, à un autre lignage que le fils de sa sœur, le souverain les aurait également peut-être classés hors de sa parentèle. On comprendrait alors pourquoi les huaccha concha « aunque sean hijos del Ynga [...] no son tenidos ni acatados ninguno destos ansi hombres como mujeres de los demás señores si no como por un orejón de los otros comunes » (Betanzos, ibid.). La dénomination de Vicaquirau ne serait d'ailleurs peut-être pas sans rapport avec le statut particulier dévolu à ses membres. Comme mentionné précédemment, vicalvecca désignant les excréments ${ }^{22}$ et quirau la « cuna de los niños » (González Holguín 1989, p. 310), Vicaquirau pourrait se traduire par « berceau d'excréments ». Bien que les données fassent défaut pour appréhender avec précision la sémantique fécale dans le contexte inca, l'assimilation des membres de l'ayllu à des "selles » dont celui-ci serait le «berceau » pourrait ne pas être étrangère à la filiation paradoxale qui leur aurait été imputée : symboliquement, l'absence théorique d'un père ne supposerait-elle pas un enfantement par une autre voie que celle de la génération ordinaire ? Zuidema proposait, pour sa part, une autre explication, de type classificatoire, qui coïnciderait plus directement avec la position cayau du groupe : selon lui, le nom de Vicaquirau assimilerait les membres de l'ayllu à la catégorie "puant» (Zuidema 1986, p. 54), une classification qu'il associe ailleurs aux « étrangers au lignage », justement couramment classés comme cayau (Zuidema 1989, p. 99). 
Betanzos ne fournit aucune précision sur l'identité du souverain dont les Vicaquirau auraient constitué la classe de descendants la moins considérée. En admettant, comme le suggère l'étroite cohérence avec les données concernant les ayllu Capac et Hatun, qu'il s'agissait effectivement de Pachacuti, la descendance du souverain, couvrant les trois ayllu de Hanan Cuzco, se serait donc organisée selon le schéma suivant (Figure 7).

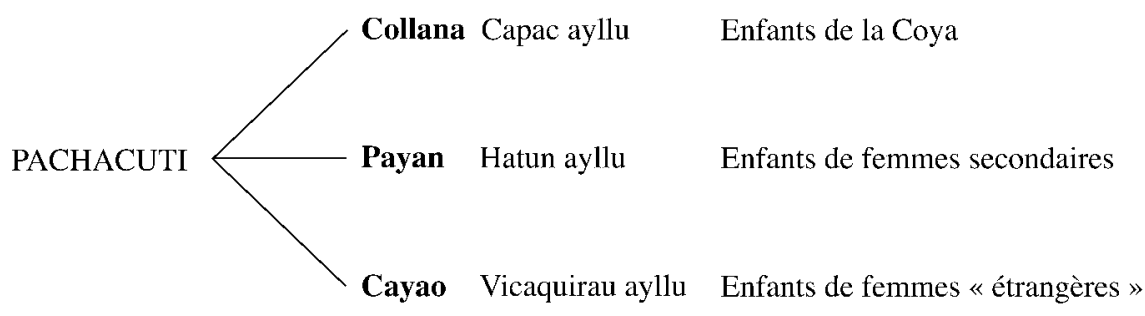

Fig. 7 - Les trois ayllu de Hanan Cuzco comme groupes de descendants de Pachacuti.

\section{Hatun AYLlu ET IÑaCa PANACA}

La double dénomination de Hatun ayllu/Iñaca panaca (ayllu) constitue assurément la manifestation la plus problématique de la dichotomie ayllu/panaca (ayllu). L'attention qu'il convient d'y porter se justifie d'autant plus que, dans ce qui pourrait être considéré comme un cas limite, réside peut-être aussi la possibilité d'une appréciation plus fine de la dichotomie elle-même.

Les rares informations concernant les femmes iñaca semblent concorder avec l'identification de Hatun ayllu comme groupe de descendants secondaires de Pachacuti. Le terme iñaca figurant dans les lexiques comme synonyme de palla (cf. note 15), " hijas de los yndios cavalleros hanancuzco hurincuzco » (Guaman Poma 1989 [1613], f. 758), les iñaca devaient occuper dans la hiérarchie féminine de Cuzco un rang inférieur à celui des ñusta, "prenzezas [...] hijas de los auquiconas, prinsepes nietos y bisnietos de los yngas »(ibid.). Si l'on en croit Garcilaso de la Vega, c'est l'ascendance maternelle qui fondait la distinction entre les ñusta, "legitimas en la sangre real », et les palla, " no legitimas en sangre " (Garcilaso de la Vega 2003, p. 79). Considérant l'identification des iñaca comme palla, le qualificatif désignerait alors les filles du souverain et de ses femmes secondaires (Zuidema 1989, p. 110), définition qui rejoint l'information de Sarmiento de Gamboa sur Hatun/Iñaca, censé regrouper les « enfants bâtards » de Pachacuti. Filles secondaires de l'Inca, les iñaca étaient apparemment destinées à des alliances matrimoniales spécifiques, notamment avec des chefs 
provinciaux (Murúa 2001 [1616], p. 366), usage rapporté par Garcilaso de la Vega selon lequel « muchas veces daban los reyes parientas suyas de las bastardas por mujeres a grandes señores » (ibid.).

Hatun/Iñaca rassemblant les descendants secondaires de Pachacuti, Iñaca panaca désignait-il alors spécifiquement le contingent féminin, Hatun ayllu qualifiant symétriquement la descendance masculine ? Certaines spécificités qui le distinguent des autres groupes aristocratiques permettent d'avancer une hypothèse quant aux raisons de cette double dénomination et qualification.

\section{Singularités statistiques}

Rapportant le contenu de la carta de poder que lui avaient adressée en 1603 les représentants de la noblesse indigène de Cuzco, Garcilaso de la Vega retranscrit les données numériques et note l'effectif de chaque groupe ${ }^{23}$ qui oscille entre 20 et 60 individus, à l'exception d'« Inca panaca » qui compte alors 99 membres. Une sur-représentation due, selon le chroniqueur, au fait que le groupe rassemblait non seulement les descendants de Pachacuti, mais aussi ceux de son fils Inca Yupanqui ${ }^{24}$. Si Garcilaso distingue à tort Pachacuti et Inca Yupanqui, sur le plan statistique sa remarque est en revanche tout à fait justifiée : la moyenne étant de 42, 54 individus par groupe, l'effectif d' « Inca panaca » (99 personnes) correspondrait effectivement au double d'un groupe de taille moyenne. Bien qu'à première vue rien n'empêche d'y voir l'effet d'un taux de fécondité particulièrement élevé, une autre source vient toutefois appuyer le caractère significatif de cette donnée statistique. En février 1572, Sarmiento de Gamboa avait fait réunir les "indios principales y de mejor entendimiento de los doce ayllus y descendencias de los doce incas " afin de vérifier sa version de l'histoire inca (Sarmiento de Gamboa 2001, pp. 172-178). La liste des principales des différents groupes est enregistrée par Alvaro Ruiz de Navamuel, l'écrivain officiel du vice-roi Toledo (ibid., pp. 174-175). Or on observe que, tandis que le nombre de principales oscille entre 2 et 4 individus, il atteint 8 personnes pour Hatun/Iñaca, un effectif qui cette fois dépendait moins de facteurs démographiques que structurels, inhérents à l'organisation interne des groupes.

\section{Particularités de l'implantation territoriale}

Hatun/Iñaca se démarque également des autres groupes aristocratiques sur le plan de la propriété terrienne. Alors que ces derniers tendaient à occuper des territoires continus ${ }^{25}$, la documentation d'archives concernant l'implantation territoriale de Hatun/Iñaca témoigne d'une toute autre configuration (Figure 8).

En 1575, les ayllu Hatun et Capac demandent à se voir accorder officiellement la possession de "Yuncaypampa, Chuquipampa, Tambomachay y Yaullicancha, Corobillca, Rocacancha, Sonchocatta, Guaynacalla » qu'ils occupaient jusqu'alors 
sans titre de propriété (BNP, B561, 1685, f. 23v). Si l'on ne peut localiser précisément l'ensemble des terres mentionnées ${ }^{26}$, le document fournit cependant la liste des bornes qui délimitaient l'ensemble ${ }^{27}$. Pour autant qu'on puisse en juger, le territoire des ayllu couvrait une vaste superficie au nord-est de Cuzco : apparemment bordé à l'ouest par le chemin de Curimarca qui, partant de Cuzco près du Sacsayhuaman, passait par Kenko et Puca Pucara, il s'étendait au nord jusqu'à Macaicalla (Cobo 1956, p. 176), une plaine correspondant à l'actuel Qeser Qasa (Bauer 2000, p. 89), et à l'est jusqu'aux environs de Callachaca. La documentation indique que les Capac et Hatun occupaient cet espace avant la création en 1560 des premières paroisses de Cuzco : en 1552, Charles Quint avait accordé par cédule royale à des " nietos legitimos por linea recta del gran Topa Ynga Yupangui » la possession d'une surface de terres au nord de Cuzco. Dans cet ensemble figurait une parcelle à Macaicalla « que linda [...] para abajo con las tierras de los yngas de Capac ayllo Hatun ayllu » (AHC, Real Audiencia, Otras Audiencias, leg. 183, 1694-1782, f. 3v, publié dans Rostworowski 1962, p. 161).

Quelques documents concernant spécifiquement Hatun ayllu suggèrent son implantation dans la partie orientale du territoire délimité en 1575. En 1574, Beatriz Cusirimay Coya, épouse de Diego Cayo, l'un des chefs de Hatun ayllu ${ }^{28}$, vend au nom de celui-ci une parcelle " en el valle desta ciudad donde dizen Rucre » (AHC, Notariales, prot. 22, 1573-1574, f. 374), jouxtant la propriété de Juan Quispicusi, autre membre éminent de l'ayllu ${ }^{29}$. Rucre correspond à l'actuel Lucrepata, un quartier au nord-est de Cuzco, dans la paroisse de San Blas où Diego Cayo avait sa demeure (Cobo 1956, p. 175). Quelques années plus tard, son fils Diego Tupa Yachi vend à un Espagnol une vaste surface de terre héritée de son père " en lo alto de san Blas camyno de la provincia de los andes junto al cerro llamado amaro » (AHC, Notariales, prot. 10, 1584, f. 292). Bordés par le chemin de l'Antisuyu, les terrains se trouvaient au nord de Rucre, à proximité d'Amaru, toponyme désignant à la fois une montagne et un ensemble archéologique qui correspondrait à la huaca d'Amaromarca huasi, " en el camino de los Andes » (Cobo, ibid.), à l'ouest de Callachaca. Dans cette zone entre Rucre et Callachaca se trouvait Patallacta, une " maison » qui aurait abrité la momie de Pachacuti Inca Yupanqui avant son transfert à Tococachi (San Blas) où Polo de Ondegardo la découvre en 1559 (Betanzos 1987, p. 149 ; Cobo ibid., p. 169 ; Sarmiento de Gamboa 2001, p. 127). L'implantation de Hatun ayllu y est attestée jusqu'en 1786 : à cette date, la délimitation des terres de Patallacta précise qu'une des bornes se trouvait « donde esta una acequia de agua que biene del paraje nombrado Ucu Ucu y una peña viva donde se dividen las tierras de los yndios de Atun ayllu » (AHC, Notariales, prot. 120, 1827-1830, f. 289).

Dans aucun document concernant ces terres Hatun ayllu ne figure sous le nom d'« Iñaca panaca ». La documentation atteste cependant de l'existence de terrains appartenant nommément à Iñaca panaca, mais, cette fois, dans la paroisse de San Jerónimo, au sud de la vallée, dans le secteur de Collasuyu. En 
1595, le licenciado Francisco de Loayza déclare « vacants » les terrains d'Oscollopampa, en bordure du Huatanay, afin de pouvoir les vendre au profit de la couronne espagnole. Le document précise que les limites des parcelles, précédemment propriété du capitaine Garcilaso de la Vega - le père du chroniqueur - « de ancho son por una parte tierras de los yndios de nacapanaca » (AHC, Colegio de Ciencias, leg. 9, cuad. 3, 1590, f. 4). « Naca panaca » est une altération d'« Iñaca panaca ", comme l'atteste un document daté de 1630 mentionné par Rostworowski et qui confirme cet emplacement (Rostworowski 1983, p. 144).

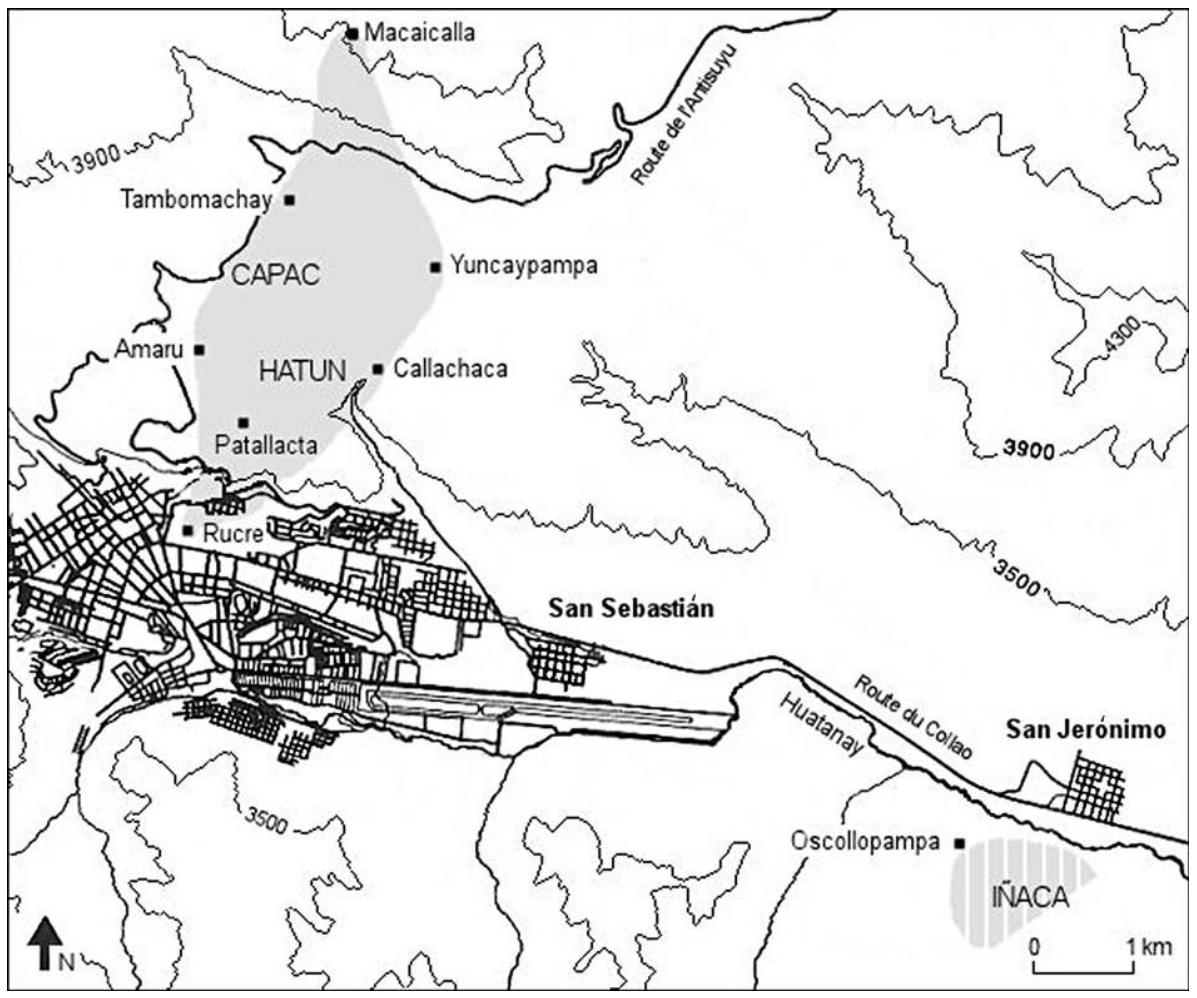

FIG. 8 - Reconstitution approximative des territoires de Hatun ayllu et Iñaca panaca (ayllu) $\mathrm{XVI}^{\mathrm{e}}$ siècle (carte d'après Bauer 2000).

\section{Hatun et Iñaca, ayllu et panaca (ayllu)}

$\mathrm{Au}$ vu de l'ensemble de ces données qui le distinguent nettement des autres groupes aristocratiques par de multiples effets de dédoublement, la solution la plus plausible au problème de la double dénomination et qualification du groupe 
ne serait-elle pas alors d'envisager, avec Rostworowski, que Hatun ayllu et Iñaca panaca constituaient en réalité deux entités distinctes (ibid.), réduites à l'unité par l'équation supposée entre un ancêtre royal et un groupe de descendants ? Comme le suggère le terme iñaca, cette différenciation aurait pu se fonder sur un critère sexuel, Iñaca panaca ayant alors regroupé à l'origine les " filles bâtardes » (Sarmiento de Gamboa 2001, p. 126) de Pachacuti, et Hatun ayllu les « enfants mâles » (ibid.) du souverain et de ses femmes secondaires. Au final, en admettant l'hypothèse avancée plus haut sur Vicaquirau ayllu, il faudrait dès lors compter quatre groupes de descendants de Pachacuti, répartis en trois classes en fonction du statut de leurs mères (Figure 9).

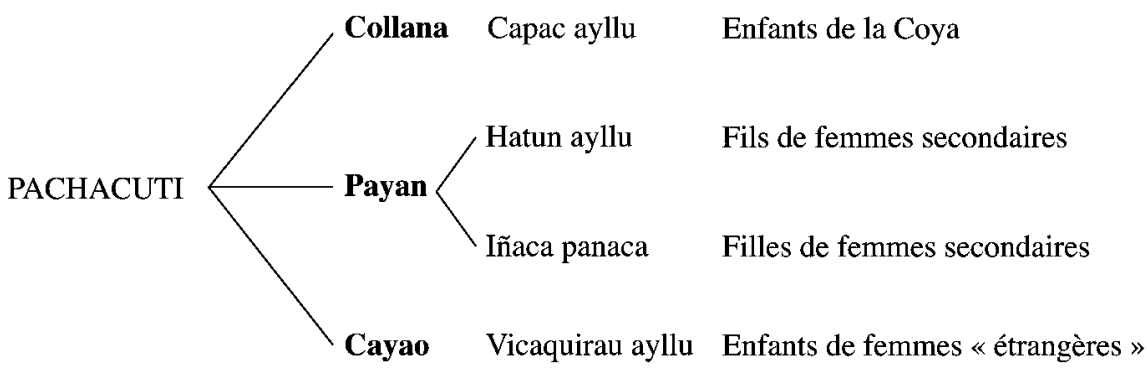

FIG. 9 - Diagramme hypothétique de la descendance de Pachacuti.

Si elle est exacte, la distinction entre Hatun ayllu et Iñaca panaca peut s'avérer précieuse pour préciser la différence entre ayllu et panaca (ayllu). En effet, la pérennité même d'Iñaca panaca supposerait que les enfants des filles secondaires de Pachacuti étaient affiliés au groupe de leurs mères. Dans la mesure où ils se considéraient comme descendants du souverain et rendaient un culte aux lieux associés à sa mémoire ${ }^{30}$, leur affiliation ne pourrait s'expliquer simplement par une résidence matrilocale, mais plus certainement par un principe matrilinéaire. À l'inverse, Hatun ayllu, rassemblant les enfants des fils secondaires de Pachacuti, témoignerait d'un fonctionnement patrilinéaire. Sous réserve de validation par une analyse poussée des autres groupes aristocratiques, on en reviendrait ainsi aux propositions développées par Zuidema quant à la connotation patrilinéaire de l'ayllu et matrilinéaire de la panaca (ayllu), quoique par d'autres voies et, surtout, dans une perspective distincte : non pas celle d'une appréciation variable sur un même goupe, considéré suivant les circonstances comme patri- ou matrilinéaire, mais bien d'une nette différenciation entre deux types de groupes sociaux présentant des modes de filiation distincts.

$\mathrm{S}$ 'il reste évidemment à déterminer les raisons d'une telle distinction au sein des descendants secondaires de Pachacuti, il conviendrait également d'expliquer 
une différence de traitement manifestée notamment par la mention du seul Hatun ayllu lors de la course rituelle de la Situa qui, contrairement à Iñaca panaca (ayllu), devait bénéficier d'un ceque propre, omis par le rédacteur de la Relación de las huacas. Quelles que soient les raisons de cette inégalité de traitement, on observe toutefois que, dans ce cas précis, elle vient opportunément rétablir une harmonie formelle : en excluant Iñaca de la projection spatiale de la structure sociale, les deux moitiés Hanan et Urin retrouvent une parfaite symétrie, chacune se trouvant alors composée de trois ayllu et de deux panaca.

\section{CONCLUSION}

Si le réexamen attentif des sources classiques et l'apport de la documentation d'archives permettent de mettre en évidence un clivage typologique au sein des groupes aristocratiques cuzquéniens, il reste à en définir plus précisément les termes. En effet, si une différence hypothétique dans le mode le filiation pourrait constituer un premier élément de distinction, la dichotomie ayllu/panaca (ayllu) ne s'y réduit visiblement pas. Comme pour Hanan Cuzco, certains indices suggèrent également l'existence d'un lien structurel entre les trois ayllu d'Urin Cuzco. Entre les ayllu Apomayta et Uscamayta, le partage du terme mayta - couramment utilisé dans les noms de leurs membres, comme d'ailleurs chez ceux de Hahuaynin ayllu ${ }^{31}$ - vient se doubler de la connotation classificatoire d'apu (« chef») et usca ("mendiant») que Zuidema a rapprochés de collana ("principal, cosa excelente») et de huaccha ("pauvre»), qualifiant des groupes en position cayau (Zuidema 1995 [1964], p. 219), comme semblaient déjà l'illustrer les huaccha concha de l'ayllu Vicaquirau. Enfin, le nom de Hahuaynin dérive de hahua ("petit-fils»), associé à une position payan dans certaines tripartitions exprimées dans une terminologie proche de celle des ayllu d'Urin Cuzco, comme la classification « Capac Inca, Hahua Inca, Huaccha Inca » évoquée par Guaman Poma de Ayala (1989, f. 244). Le problème des panaca offre plus de résistance à l'analyse, non seulement du fait du manque de documents suffisamment explicites pour éclairer leur fonctionnement, mais aussi, contrairement aux ayllu, par la difficulté à les rapprocher d'institutions andines connues. À première vue, la seule organisation féminine pouvant offrir une perspective analogique serait celle des aclla, "femmes choisies» auxquelles Polo de Ondegardo associait son " apu panaca », et dont on sait que, comme les iñaca, elles participaient d'un dispositif d'alliances politiques par voie matrimoniale. Cependant, si l'existence de quatre classes d'aclla dont témoigne la plupart des sources peut suggérer une correspondance formelle avec les quatre panaca dont l'origine historique n'est pas directement repérable, l'existence d'Iñaca panaca (ayllu) affaiblit ce parallélisme comme elle perturbait déjà la trop belle symétrie des moitiés Hanan et Urin. 
Une caractérisation plus nette des ayllu et panaca cuzquéniens ainsi que les modalités de leur intégration dans un ensemble organisé nécessitent donc une recherche approfondie, notamment par le recours à des fonds documentaires encore trop peu exploités. L'analyse embryonnaire qui précède autorise toutefois à dresser un constat général qui constraste avec la radicalité de certaines postures théoriques : ni agrégat de segments homogènes juxtaposés par le temps, ni totalité organique plus ou moins soustraite à l'histoire, la société cuzquénienne semble traversée par des lignes de partage ordonnées par un jeu de symétrie peut-être forcé qui, à terme, laissent entrevoir autant le passage de l'histoire que des effets de structure persistants.

Parce qu'elle met en question un lien supposé nécessaire avec la structure sociale, la partition des groupes aristocratiques permet également d'envisager le récit dynastique inca comme un champ d'analyse autonome, dégagé de l'alternative intenable entre le mythe pur et la stricte historicité au profit d'une réalité plus complexe. De ce point de vue, les indices touchant au fonctionnement des trois ayllu de Hanan Cuzco autorisent certaines observations. Ainsi, l'historicité parfois contestée de Pachacuti Inca Yupanqui semble s'affermir par le repérage d'une multiplicité de groupes de descendants. En comptant parmi eux l'ayllu Vicaquirau, Inca Roca perdrait au contraire de sa substance, son statut d'ancêtre tutélaire pouvant alors apparaître comme une convention contemporaine ou postérieure au règne de Pachacuti, souverain réformateur auquel la tradition orale imputait justement la composition ou la réélaboration de l'histoire dynastique. De même, en admettant la possibilité d'une analogie entre les trois ayllu de chaque moitié, au moins deux des trois ancêtres royaux des ayllu d'Urin Cuzco auraient alors pu être imposés à une infrastucture sociale préexistante. C'est alors sur le sens d'un tel dispositif et les raisons qui le rendaient souhaitable ou nécessaire qu'il conviendrait de s'interroger. Au-delà des possibilités qu'elle offre pour l'étude de la société inca dans l'espace de Cuzco, la distinction entre ayllu et panaca (ayllu), par la brèche qu'elle ouvre entre la structure sociale et l'histoire dynastique, incite donc à questionner non plus tant la nature de l'histoire dynastique que son utilité sociale et une vocation dont on peut d'ores et déjà suspecter le caractère éminemment politique. *

* Manuscrit reçu en novembre 2011, accepté pour publication en octobre 2012.

\section{Notes}

Je remercie chaleureusement Pablo Sendón pour ses commentaires et conseils avisés, ainsi qu'Éric Taladoire pour sa relecture attentive.

1. Diego Fernández (1963 [1571], vol. II, p. 84) fournit sa liste des " parcialidades y linages », en s'appuyant apparemment sur Las Casas (Julien 2000, p. 84). Román y Zamora (1897 [1575], vol. II, p. 26) reprend également la liste de Las Casas, avant d'être copié à son tour par Gutiérrez de Santa 
Clara (Pärssinen 2003, p. 67). Murúa (1946 [ca 1609], pp. 79-80) donne pour sa part une liste apparemment inspirée de Fernández (Pärssinen ibid., pp. 58-59).

2. Comme le rappelle Pärssinen (ibid., p. 55), les deux dominicains entretenaient une relation épistolaire, Santo Tomás constituant l'une des principales sources d'informations péruviennes de Las Casas. Certaines de ces lettres ont été conservées à l'Archivo General de Indias (par exemple AGI, Patronato 252, Ramo 22). On sait par ailleurs que Santo Tomás avait écrit, à la fin des années 1540, une Relación de las antigüedades del Perú, aujourd'hui disparue (Araníbar in Pachacuti Yamqui 1995, p. 316).

3. Selon ce modèle bien connu, la progéniture d'un souverain disparu formait un groupe de descendants jouissant des biens accumulés par le défunt, également destinés à entretenir sa mémoire et alimenter sa momie en offrandes provenant des terres qu'il avait conquises. L'héritier au trône, exclu de cet héritage, devait à son tour constituer son propre lignage et accumuler un patrimoine personnel (voir par exemple Cieza de León 1996 [1553], pp. 28-29, Polo de Ondegardo 1990 [1571], p. 110, également Conrad et Demarest 1984).

4. "El barrio y parte de Hanan Cuzco, que era principal, subdividió en cinco barrios o partes : al uno y principal nombró Capac ayllo, que quiere decir "el linaje del Rey", con éste juntó gran multitud de gente y parte de la ciudad, que fuesen de aquel bando; al segundo llamó Iñaca panaca ; el tercero Cuçco panaca ; el cuarto Aucaylli panaca ; el quinto Vicaquirau panaca » (Las Casas 1967, p. 581).

5. De ce point de vue, le cas de Sarmiento de Gamboa peut sembler symptomatique, le chroniqueur du vice-roi ayant apparemment choisi d'éluder les problèmes terminologiques en attribuant le qualificatif panaca à l'ensemble des groupes, à l'exception de Hatun ayllu et Capac ayllu. Cet état de fait pourrait également expliquer l'attribution ponctuelle du qualificatif panaca à des groupes unanimement reconnus par ailleurs comme des ayllu, par exemple l'ayllu non aristocratique Masca, nommé par Molina « Masca panaca ayllu » (1989 [1575], p. 75), peut-être du fait qu'il était cité à la suite de «Chima panaca ayllu » et «Raura panaca ayllu ». Panaca s'est également vu parfois associé par erreur à des noms de personnes, en particulier à l'un des chefs de l'ayllu Uscamayta, Juan Tambo Uscamayta (Sarmiento de Gamboa 2001, p. 69), rebaptisé « Juan Tambo Maytapanaca » par Acosta (1954 [1590], p. 203), reprenant une erreur de Polo de Ondegardo ou de son scribe remontant à l'année 1560 (Rowe 2003b, p. 136).

6. Pachacuti Yamqui qualifie les « orejones », descendants des souverains Incas, de " mancopchurin cuzco » («Cuzco(s) fils de Manco [Capac]»), qu'il oppose à d'autres «Cuzcos »: " cacacuzcos y ailloncuzcos, que son caballeros particulares » (Pachacuti Yamqui 1995 [1613], f. 40).

7. Ce groupe, également mentionné par Garcilaso de la Vega, était censé rassembler les descendants de Huayna Capac (2003 [1609], p. 730). Cependant, Tomebamba (Tumipampa) n'apparaît pas dans les chroniques antérieures aux années 1570 et, comme le notait Bauer, aucun ceque ne lui est attribué dans la Relación de las huacas (Bauer 2000, p. 46). Il n'est par ailleurs jamais mentionné dans l'abondante documentation d'archives que nous avons pu consulter concernant la propriété terrienne des groupes aristocratiques cuzquéniens (Segalini 2009). Villanueva Urteaga avait trouvé des documents témoignant en revanche de l'existence d'un ayllu Tomebamba dans la vallée de Yucay, rassemblant des personnes d'origines diverses chargées de cultiver des terres destinées à alimenter la momie de Huayna Capac (Villanueva Urteaga 1970, p. 4.), mais il ne s'agissait en aucun cas de descendants du souverain. Faut-il alors supposer que, pour des raisons qui restent à élucider, certains descendants de l'Inca auraient repris à leur compte le nom de ce groupe au début de la période tolédane?

8. Voir Guaman Poma de Ayala 1989 [1613], f. 253, Polo de Ondegardo 1916 [1559], p. 23 et Betanzos 1987 [1551], p. 72.

9. Voir Zuidema 1995 [1964], p. 74 et Bauer 2000, p. 46. Ce que confirme Polo de Ondegardo, précisant qu'au terme de la course rituelle de la Situa « se hazía el lavatorio general en los arroyos y fuentes cada uno en su seque y pertenencia " (Polo de Ondegardo 1916, p. 23 [souligné par l'auteur, L. S.]). Rappelons que les ceque, « rayons » ou « lignes » en quechua (González Holguín 1989 [1608], p. 81), constituaient des lignes imaginaires (au nombre de 41) irradiant théoriquement depuis le 
Coricancha et couvrant la vallée de Cuzco et au-delà, reliant entre eux des lieux sacrés (fontaines, pierres, bâtiments...) dénommés huaca.

10. Une partie de cette documentation a été publiée dans Segalini 2009.

11. Voir notamment Rowe 1985, Sherbondy 1986 et Zuidema 1983.

12. «Panaca, o panari, o panatacri. Y aunque 》 (González Holguín 1989 [1608], p. 277).

13. Par exemple : « Sanctissimo apu capac panaca yayanchic Paulo, hina sutiyoc, summo Pontificecunap pichcañequenmi » (Pérez Bocanegra 1631, p. 497) et "Tucui atipac, viñaicac Dios, yanaiqui apupanacaicu N. sumo Pontifice ñisca [souligné par l'auteur, L. S.] » (ibid., pp. 535-536). Nous tenons à remercier ici César Itier qui a attiré notre attention sur l'ouvrage de Pérez Bocanegra.

14. " Huc ayllulla .I. huc panacalla, son todos los que son de una familia, y casta " (ibid., p. 613). Suivi du suffixe manta, indiquant une origine ou une appartenance, il l'utilise également pour exprimer l'affiliation de deux contractants fictifs d'un mariage, « Pedro » de l'ayllu Salloc et « Maria » de l'ayllu Antahuayla : "Pedro N. Cari [N. cari], salloc panacallamanta, Maria. N. huarmiri Antahuailla Ayllu panacamanta » (ibid., p. 619).

15. «Iñaca : la mantellina de la cabeza ; Yñacca ñusta : la señora de ayllo de Incas, o noble; Iñaca yñacalla pachallicuni o palla pallalla : vestirse galanamente la muger muy pintada » (González Holguín 1989 [1608], p. 368) ; " Iñaca, vel Palla: Muger que viene de casta noble de los Ingas. Iñacachasitha: Vestirse al modo destas mugeres. Y hazer muy de la señora » (Bertonio 1984 [1612], $2^{\mathrm{e}}$ partie, p. 175). "Palla o ygñaca : dama, es casi señora » (Santo Tomás 1951 [1560], p. 335).

16. " Ayllo. Parcialidad genealogía linage, o parentesco, o casta. Ayllo. El genero, o especie en las cosas. Ayllo, o riui : Bolillas assidas de cuerdas para trauar los pies en la guerra, y para caçar fieras, a aues y tirar a trauar pies o alas » (González Holguín 1989, p. 39).

17. Alonso Enríquez de Guzmán (1538-1540) en donne une description précise : " [Usan de unas] armas que se llaman ayllos, que son desta manera : tres piedras redondas, metidas y cosidas en unos cueros a manera de bolsas puestas en unos cordeles con tres ramales, a cada cabo de cordel puesta su piedra, de largos de una braza, todo uno " (cité dans Porras Barrenechea 1986, p. 162). Si la description de Cristóbal de Albornoz (1582) diffère quelque peu quant aux matériaux utilisés, la structure générale est en tout point semblable : " (a) yllos, que son tres ramales de soga hecha de niervos de animales o de cueros de ellos, a los cavos unas pelotas de plomo. » (Albornoz 1989, p. 175).

18. Au milieu du XVIII ${ }^{\mathrm{e}}$ siècle, une certaine Antonia Cuba réclame la possession de parcelles situées à Machacapirca, dans la paroisse de San Blas, au titre de «desendiente de sangre del Gran Tupa Yupanqui y de Amaro Topa señores que fueron de estos reynos en tiempo de la gentilidad " (AHC, Intendencia, leg. 2, 1784-1786, exp. 1, f. 67v). Afin d'appuyer sa demande, elle présente des documents remontant aux années 1560 à 1592, parmi lesquels un document de 1561 où il apparait que les terrains en question avaient déjà été réclamés par "Pisatopa, y don Juan, don Garcia y los demas minores desendientes de Amaro Topa Ynga » (ibid., f. 39). « Pisatopa » désigne ici un chef de l'ayllu Payan de Capac ayllu, baptisé sous le nom de Cristóbal, dont on retrouve la trace une vingtaine d'années plus tard dans un autre procès touchant une fois encore à la propriété de Machacapirca : « Don Francisco Pilcotopa en nombre de don Juan Tito Topa, don Garcia y don Christoval Pisatopa [...] hijos y nietos de Amaro Topa Ynga » (ibid. f. 48v). Grâce à la documentation réunie par Antonia Cuba, on connaît d'ailleurs le nombre de membres du lignage bénéficiant à l'époque du partage foncier : « de los hijos de Amaro Topa Ynga [... ] fueron veinte y siete o veinte y ocho entre todos los yngas barones y mugeres a quien se les dio posesion » (ibid., f. 44).

19. À côté de Topa Inca Yupanqui et d'Amaro Topa Inca, Sarmiento de Gamboa mentionne « dos hermanos de Tupac Inca, nombrados el uno Auqui Yupanqui y el otro Tilca Yupanqui " (Sarmiento de Gamboa 2001, p. 118). Tilca Yupanqui aurait été tué par l'un de ses frères au cours d'une campagne militaire dans le Chinchaysuyu (ibid., p. 125), ce qui ne laisse au final que trois fils légitimes. On pourrait alors proposer d'identifier Auqui Yupanqui avec Tupa Yupanqui, ancêtre de la section Cayau de Capac ayllu, qui n'apparaît pas chez Sarmiento de Gamboa, du moins sous ce nom. La mention de trois fils légitimes s'accorde cependant trop parfaitement avec la tripartition collana, payan, cayau pour ne pas envisager la possibilité d'une fratrie plus importante. 
20. Contrairement aux autres sources, Pérez Bocanegra, probablement inspiré par la parenté européenne, emploie concha pour désigner indifféremment les enfants du frère ou de la sœur d'un homme, tout en l'utilisant par ailleurs comme un équivalent de « cousin », parallèle ou croisé (Pérez Bocanegra 1631, p. 610).

21. "Guaccha: pobre varon o muger; Guaccha: huérfano»(Santo Tomás 1952, p. 280); " Huaccha : pobre o huérfano » (González Holguín 1989, p. 167).

22. "Vecca : la suciedad de pança y tripas » (ibid., p. 350).

23. "Chima panaca: son cuarenta [...] Raurava panaca: son sesenta y cuatro [...] Hahuanina ayllu : son sesenta y tres [...] Apu Maita : son cincuenta y seis [...] Usca Maita : son treinta y cinco [...] Uicaquirau : son cincuenta [...] Ailli panaca : son cincuenta y uno [...] Zoczo panaca : son sesenta y nueve [...] Inca panaca [...] son noventa y nueve [...] Capac Aillu [...] no son mas de diez y ocho" (Garcilaso de la Vega 2003, p. 730).

24. "A la descendencia del Inca Pachacutec y a la de su hijo Inca Yupanqui, juntándolas ambas, llaman Inca Panaca, y así es doblado el número de los descendientes, porque son noventa y nueve " (Garcilaso de la Vega, ibid.).

25. Voir Segalini 2009

26. La Relación de las huacas permet néanmoins de localiser certaines d'entre elles. Yuncaypampa y est décrit comme une plaine bordant le chemin des Andes (Antisuyu) (Cobo 1956, p. 176), correspondant à l'actuel Yuncaypata, à l'ouest du chemin vers Pisac (Bauer 2000, pp. 92-93). La plaine de Chuquibamba se situe à proximité du Sacsayhuaman (Cobo ibid., p. 170), probablement entre la forteresse et le site de Kenko Chico (Bauer ibid., p. 59). Tambomachay, neuvième huaca du premier ceque d'Antisuyu " en un cerro cerca del camino de los Andes » (Cobo ibid., p. 175), correspond vraisemblablement au complexe archéologique de Puca Pucara (Niles 1987, p. 180). Quant à Corovilca, il apparaît mentionné dans la Relación comme une carrière de pierres sur le deuxième ceque d'Antisuyu (Cobo ibid., p. 176).

27. "Empiezan desde un mojon de piedra llamada Rumi hurconca y de alli ba a dar a otro mojon llamado Yllapasca cancha y de alli a otro mojon que se llama rocacancha y por la parte de abajo con tierras $y$ de Thomas y Andres Narbaes mulattos y mojon llamado condorconca y ba por el camino biejo del ynga hasta dar en el camino rreal que ba a curimarca y por el dicho camino hasta dar a una peña que llaman Guayrac machay y de alli derecho por el propio camino que ba a curimarca hasta camaypatta y de alli guayllay cocha y de alli una alberca llamada chullquicocha y de alli ba a dar otro mojon por una quebradilla arriba por donde alindan desde las tierras de doña luisa y sus hermanos hasta en arroyo de Tambomachay y con tierras de Francisco de Villacastin y Juan Balca y con tierras de Geronimo Costilla y de alli con los mojones llamados chuquiguaylla patan tioc macha y macaicalla casa. " (BNP, B561, 1685, f. 25v-26).

28. "Los que sustentan ahora este linaje, que son vivos, son Don Diego Cayo, Don Felipe Ynquil, Don Juan Quispicusi, Don Francisco Chalco Rimachi, Don Juan Illac. » (Sarmiento de Gamboa, 2001, p. 126 [souligné par l'auteur, L. S.]).

29. " Alinda con chacara de mi el dicho Juan Quispicusi » (AHC, Notariales, prot. 22, 1573-1574, f. 374); Sarmiento de Gamboa ibid.

30. Ils rendaient par exemple un culte à la huaca de Cusicancha, lieu de naissance supposé de Pachacuti : "Cusicancha era el lugar donde nació inca yupanqui, frontero del templo de coricancha, y por esta razón ofrecían allí los del ayllu inacapanaca » (Cobo 1956, p. 171).

31. Voir par exemple les noms des principales des différents groupes enregistrés par Sarmiento de Gamboa (ibid., pp. 66, 69 et 174). 


\section{RÉFÉRENCES CITÉES}

ABRÉVIATIONS UTILISÉES (FONDS D'ARCHIVES)

Archivo General de Indias (AGI), Séville.

Archivo Histórico del Cuzco (AHC), Cuzco.

Biblioteca Nacional del Perú (BNP), Lima.

Acosta José (de)

1954 Historia natural y moral de las Indias, in Obras completas, Ediciones Atlas, coll. « Bibliotheca de autores españoles » 73, Madrid [1590].

Albornoz Cristobal (de)

1989 «Instrucción para descubrir todas las guacas del Pirú y sus camayos y haziendas », in Henrique Urbano et Pierre Duviols (éd.), Fábulas y mitos de los incas, Historia 16, Madrid, pp. 161-198 [1582].

BAUER Brian

2000 El espacio sagrado de los incas. El sistema de Ceques del Cuzco, CBC, Cuzco.

BERTONIO Ludovico

$1984 \quad$ Vocabulario de la lengua aymara, CERES/IFEA/MUSEF, Cochabamba [1612].

BETANZos Juan (de)

1987 Suma y narración de los incas, María del Carmen Martín Rubio (éd.), Atlas, Madrid [1551].

Cerrón Palomino Rodolfo

1998 «El cantar de Inca Yupanqui y la lengua secreta de los incas », Revista andina, 32, pp. 417-452.

Cieza de León Pedro

1996 Crónica del Perú, Segunda parte, Pontificia Universidad Católica del Perú, Lima [1553].

Сово Bernabé

1956 Historia del Nuevo Mundo in Obras, tome II, Ediciones Atlas, coll. « Bibliotheca de autores españoles » 92, Madrid [1653].

CONRAD Geoffrey et Arthur Demarest

1984 Religion and Empire. The dynamics of Aztec and Inca expansionism, Cambridge University Press, Cambridge.

Declaración

1920 «Discurso sobre la descendencia y gobierno de los Incas » [Declaración de los quipocamayos a Vaca de Castro], in Horacio Urteaga et Carlos Romero (éd.), Colección de libros y documentos referentes a la historia del Perú, $2^{\mathrm{e}}$ série, tome III, Informaciones sobre el Perú antiguo, Imprenta y Librería Sanmartí, Lima, pp. 3-53 [1608 (1542)].

Duviols Pierre

1979a «La dinastía de los incas. ¿ Monarquía o diarquía? Argumentos heurísticos a favor de una tesis estructuralista », Journal de la Société des Américanistes, 66, pp. 67-83. 
1979b «Datation, paternité et idéologie de la "Declaración de los Quipucamayos a Vaca de Castro" ", in Les cultures ibériques en devenir, essais publiés en hommage à la mémoire de Marcel Bataillon, Fondation Singer-Polignac, Paris, pp. 583-591.

1997 «Del discurso escrito colonial al discurso prehispánico: hacia el sistema sociocosmológico inca de oposición y complementaridad », Bulletin de l'Institut français d'études andines, 26 (3), pp. 279-305.

FERNÁNDEZ Diego

1963 Primera y segunda parte de la historia del Perú, Ediciones Atlas, coll. « Bibliotheca de autores españoles », tomes CLXIV-CLXV, Madrid [1571].

Garcilaso de la Vega Inca

2003 Comentarios reales de los incas, Mercedes López-Baralt (éd.), EspasaCalpe, Madrid [1609].

Germonius Anastasius

1623 De Sacrorum Immunitatibus, in Ópera Omnia, tome I, Haeredis Bartholomaei Zannetti, Rome [1591].

GonzÁLEZ Holguín Diego

1989 Vocabulario de la lengua general de todo el Perú, llamada lengua qquichua o del Inca, Universidad Nacional Mayor de San Marcos, Lima [1608].

Guaman Poma de Ayala Felipe

1989 Nueva Corónica y Buen Govierno, Institut d'Ethnologie, Paris [1613].

HERNÁNDEZ Astete Francisco

2008 «Las panacas y el poder en el Tahuantinsuyo », Bulletin de l'Institut français d'études andines, 37 (1), pp. 29-45.

JuLIEN Catherine

$2000 \quad$ Reading Inca History, University of Iowa Press, Iowa City.

KIRCHHOFF Paul

1949 "The social and political organization of the Andean peoples », in Julian H. Steward (éd.), Handbook of South American Indians, vol. V, The comparative ethnology of South American Indians, Smithsonian Institution (Bureau of American Ethnology, Bulletin 143 [2]), Washington DC, pp. 293-311.

LAS CASAs Bartolomé (de)

1967 Apologética historia sumaria, Edmundo O'Gorman (éd.), Universidad Nacional Autónoma de México, Mexico [1562-1564].

Molina Cristóbal (de)

1989 "Relación de las fábulas y ritos de los incas », in Henrique Urbano et Pierre Duviols (éd.), Fábulas y mitos de los incas, Historia 16, Madrid, pp. 47-134 [1575].

Murúa [Morúa] Martin de

1946 Historia del origen y genealogía real de los reyes incas del Perú (ms. Loyola), Constantino Bayle (éd.), CSIC/Instituto Santo Toribio de Mogrovejo, coll. « Biblioteca Missionalia Hispanica », 2, Madrid [1609]. 
2001 Historia general del Perú (ms. Wellington), Manuel Ballesteros (éd.), Dastin, coll. « Crónicas de América », Madrid [1616].

NiLes Susan

1987

Callachaca: style and status in an Inca community, University of Iowa Press, Iowa city.

Pachacuti YamQui Juan de Santa Cruz

1995 Relación de las antigüedades deste reino del Perú, Carlos Aranibar (éd.), Fondo de Cultura Económica, Lima [1613].

PÄRSSINEN Martí

2003 Tawantinsuyu. El Estado inca y su organización política, IFEA/PUCP, Lima.

PeAse Franklin

1995 Las crónicas y los Andes, PUCP, Lima.

PÉREZ Bocanegra Juan

$1631 \quad$ Ritual formulario e institución de cura para administrar a los naturales de este reyno, Gerónymo de Contreras, Lima.

Polo DE ONDEGARdo Juan

1916 «Los errores y supersticiones de los indios sacadas del tratado y averiguación que hizo el licenciado Polo », in Horacio Urteaga et Carlos Romero (éd.), Colección de libros y documentos referentes a la historia del Perú, $1^{\text {ère }}$ série tome III, Informaciones acerca de la religión y gobierno de los Incas (1. ${ }^{a}$ parte) seguidas de las Instrucciones de los Concilios de Lima, Imprenta y Librería Sanmartí, Lima [1559].

1990 « Notables daños de no guardar a los indios sus fueros », in Laura Gonzalez et Alicia Alonso (éd.), El mundo de los incas, Historia 16, Madrid, pp. 35-171 [1571].

Porras BarRenechea Raúl

1986 Los cronistas del Perú, Banco de crédito del Perú/Ministerio de Educación, Lima.

ROMÁN y ZAMORa Jerónimo (de)

1897 Repúblicas de Indias, idolatrias y gobierno en México y Perú antes de la conquista, Victoriano Suárez, coll. « Colección de libros raros o curiosos que tratan de América », 14-15, Madrid [1575].

RosTwOROwsKi María

1962 « Nuevos datos sobre tenencia de tierras reales en el Incario », Revista del Museo Nacional, (Lima) 21, pp. 130-164.

1983 Estructuras andinas del poder, IEP, Lima.

1988 Historia del Tawantinsuyu, IEP, Lima.

Rowe John H.

1985 «La constitución inca del Cuzco », Revista histórica, 9 (1), pp. 33-73.

2003a «Una relación de los adoratorios del antiguo Cuzco », in Rowe 2003b, pp. 181-230 [1985].

2003b Los Incas del Cusco - Siglos XV, XVII, XVIII, INC, Cuzco. 
2003c «Probanza de los incas nietos de conquistadores », in Rowe 2003b, pp. 79-116 (1986 [1569]).

Rubio CORREA Marcial

1985 Para leer el Código Civil, tome I, PUCP, Lima.

Santo Tomás Fray Domingo de

1951 Lexicon o Vocabulario de la lengua general del Perú, Instituto de Historia, Lima [1560].

1995 Grammática o arte de la lengua general de los Indios de los reynos del Perú, CERA « Bartolomé de Las Casas », Cuzco [1560].

Sarmiento de Gamboa Pedro

2001 Historia de los incas (Historia índica), Miraguano/Polifemo, Madrid [1572].

SEGALINI Laurent

2009 "Organización socio-espacial del Cuzco prehispánico: datos sobre la repartición de tierras de los grupos aristocráticos incas ", Revista andina 49 , pp. 105-133.

SHERBONDY Jeanette

1986 "Los ceques : código de canales en el cuzco incaico », Allpanchis 27, pp. 39-74.

URTON Gary

1990 The History of a myth. Pacariqtambo and the origin of the Inkas, University of Texas Press, Austin.

VALCÁRCEL Luis E.

1925 Del ayllu al imperio, Editorial Garcilaso, Lima.

Villanueva Urteaga Horacio

1970 «Documentos sobre Yucay, siglo XVI », Revista del Archivo Histórico del Cuzco 13, pp. 1-148.

ZuIDEMA Reiner Tom

1977 "The Inca kinship system : a new theoretical view », in Ralph Bolton et Enrique Mayer (éd.), Andean kinship and marriage, American Anthropological Association, Washington DC, pp. 240-281.

1983 "Hierarchy and Space in Incaic social organization", Ethnohistory, 30 (2), pp. $49-75$

1986 La civilisation inca au Cuzco, PUF, Paris.

1989 Reyes y guerreros. Ensayos de cultura andina, Fomciencias, Lima.

1995 El sistema de ceques del Cuzco. La organización social de la capital de los incas, PUCP, Lima.

2010 El calendario inca. Tiempo y espacio en la organización ritual del Cuzco. La idea del pasado, Congreso del Perú/PUCP, Lima. 
